\title{
Extension and estimation of correlations in cold dark matter models
}

\author{
F. Sylos Labini ${ }^{1,2}$ and N. L. Vasilyev ${ }^{3}$ \\ 1 "Enrico Fermi Center", via Panisperna 89 A, Compendio del Viminale, 00184 Rome, Italy \\ e-mail: sylos@romal.infn.it \\ 2 "Istituto dei Sistemi Complessi" CNR, via dei Taurini 19, 00185 Rome, Italy \\ ${ }^{3}$ Sobolev Astronomical Institute, St.Petersburg State University, Staryj Peterhoff, 198504 St. Petersburg, Russia \\ Received 15 June 2007 / Accepted 30 September 2007
}

\section{ABSTRACT}

\begin{abstract}
We discuss the large-scale properties of standard cold dark-matter cosmological models characterizing the main features of the power spectrum, of the two-point correlation function, and of the mass variance. Both the real-space statistics show a very well-defined behavior on large enough scales, for their amplitudes to become smaller than unity. The correlation function, in the range $0<\xi(r)<1$, is characterized by a typical length scale $r_{\mathrm{c}}$, where $\xi\left(r_{\mathrm{c}}\right)=0$, which is fixed by the physics of the early universe. Beyond this scale it becomes negative, going to zero with a tail proportional to $-\left(r^{-4}\right)$. These anti-correlations thus represent an important observational challenge for verifying models in real space. The same length scale $r_{\mathrm{c}}$ characterizes the behavior of the mass variance, which decays for $r>r_{\mathrm{c}}$ as $r^{-4}$, the fastest decay of any mass distribution. The length-scale $r_{\mathrm{c}}$ defines the maximum extension of (positively correlated) structures in these models. These are the features expected for the dark-matter field: however galaxies, which represent a biased field, may differ in their behaviors, which we analyze. We then discuss the detectability of these real-space features by considering several estimators of the two-point correlation function. By making tests on numerical simulations, we emphasize the important role of finite size effects, which should always be controlled for careful measurements.
\end{abstract}

Key words. cosmology: observations - cosmology: large-scale structure of Universe - cosmology: miscellaneous

\section{Introduction}

In contemporary cosmological models, the structures observed today on large scales in the distribution of galaxies in the universe are explained by the dynamical evolution of purely selfgravitating matter (dark matter) from an initial state with lowamplitude density fluctuations, the latter strongly constrained by satellite observations of the fluctuations in the temperature of the cosmic microwave background radiation. The other main observational element for understanding the large-scale structure of the universe is represented by the studies of galaxy correlations. Any theoretical model aiming to explain the formation of structures must be tested against the data provided by galaxy surveys that give the important bridge between the regimes characterized by large and small fluctuations.

Models of the early universe (see e.g. Padmanabhan 1993, and references therein) predict certain primordial fluctuations in the matter density field, defining the correlations of the initial conditions, i.e. at the time of decoupling between matter and radiation. In the regime where density fluctuations are small enough, the correlation function of the present matter density field is simply related to one describing the initial conditions. In fact, according to the growth of gravitational instabilities in an expanding universe in the linear regime, perturbations are simply amplified (see e.g., Peebles 1980, and references therein). Thus today on some large scales where the correlation function is still positive, but with $\xi(r)<1$, the imprint of primordial fluctuations should be preserved. In the region of strong non-linear fluctuations an analytical treatment to predict the behavior of the two-point correlation function has not been developed yet and, in general, one makes use of numerical simulations that provide a rich, but phenomenological, description of the structures in the non-linear regime. It is in this regime, on small enough scales, where most observations have been performed until now.

We focus here on the type of correlations predicted in the linear regime by models of the early universe. While the characterization of correlations is usually done in terms of the power spectrum of the density fluctuations a real space analysis turns out to be useful to point out some relevant features from an observational point of view (see, e.g., the discussion in Gabrielli et al. 2004).

Theoretical models of density fields of primordial matter in the expanding universe are characterized by a single welldefined length scale, which is an imprint of the physics of the early universe at the time of the decoupling between matter and radiation (see e.g. Bond \& Efstathiou 1984; Padmanabhan 1993, for a general introduction to the problem). The redshift characterizing the decoupling is directly related to the scale at which the change of slope of the power spectrum of matter density fluctuations $P(k)$ occurs; i.e., it defines the wave-number $k_{\mathrm{c}}$ at which there is the turnover of the power spectrum between a regime, at large enough $k$, where it behaves as a negative power law of the wave number $P(k) \sim k^{m}$ with $-1<m \leq-3$, and a regime at small $k$ where $P(k) \sim k$, as predicted by inflationary theories. Given the generality of this prediction, it is extremely important to look for this scale in the data.

The exact location of this scale is related to several parameters, including the cosmological ones that describe the geometry of the universe at large scales (see e.g. Padmanabhan 1993; Tegmark et al. 2004; Spergel et al. 2007, for a recent determination). We discuss in what follows that the scale $r_{\mathrm{c}}$ corresponding to the wave-number $k_{\mathrm{c}}$, in a particular variant of cold dark matter (CDM) models - the so-called $\Lambda \mathrm{CDM}$ vanilla model 
- is predicted to be $r_{\mathrm{c}} \approx 124 \mathrm{Mpc} / \mathrm{h}^{1}$. On this scale the real space correlation function crosses zero, becoming negative on larger scales. In particular the correlation function presents a positive power-law behavior on scales $r \ll r_{\mathrm{c}}$ and a negative powerlaw behavior at scales $r \gg r_{\mathrm{c}}$. Positive and negative correlations are exactly balanced in way such that the integral over the whole space of the correlation function is equal to zero. This is a global condition of the system fluctuations which corresponds to the fact that the distribution is super-homogeneous (or hyperuniform), i.e. characterized by a sort of stochastic order and by fluctuations that are depressed with respect to, for example, a purely uncorrelated distribution of matter (Gabrielli et al. 2002 - see discussion below).

Note that the scale $r_{\mathrm{c}}$ marks the maximum extension of positively correlated structures: beyond $r_{\mathrm{c}}$, the distribution must be anti-correlated since the beginning, as the evolution time was not long enough for the positive correlations to be developed. Thus this scale can be regarded as an upper limit to the maximum size of structures in the present universe. The possible discoveries of larger structures is still a challenging task for observational cosmology.

A relevant problem for measuring small-amplitude values of the correlation function, i.e. when $\xi(r)<1$, is represented by the characterization and the understanding of both the systematic biases that may affect the estimators of $\xi(r)$ and the stochastic noise which perturbs any real determination. A study of that problems can be found, for example, in Kerscher (1999) and Kerscher et al. (2000), where it is shown that in general the biases in several estimators of the two-point correlation function are not negligible. In particular when there are structures of large spatial extension inside a given sample, there can be non negligible biases affecting the determination of two-point properties. We focus here on the systematic bias related to the effect of the so-called integral constraint, which distorts any estimator of the correlation function on large scales in any given sample. The integral constraint represents an overall condition on any estimator of the correlation function, which is due to the fact that the average density, estimated in any given sample, is in general different from its average ensemble value.

Here we explicitly treat the case for the simplest estimator of the two-point correlation function, the so-called full-shell or minus estimator and illustrate the situation for the other estimators by studying artificial distributions. In particular, we devote most of our attention to the estimator introduced by Davis \& Peebles (1983), which is still very used in the literature, and to the estimator introduced by Landy \& Szalay (1993), which is the most popular one. Kerscher et al. (2000) also considered other estimators, like the Hewett estimator (Hewett 1982) and the Hamilton estimator (Hamilton 1993) and have shown that the results obtained with the Landy and Szalay estimator are almost indistinguishable from the Hamilton estimator.

In this way we will be able to identify the problems related to the identification of correlations above the mentioned scale $r_{\mathrm{c}}$. We will then propose several tests to be applied to the galaxy data, in order to define the strategy for studying the correlation function at small amplitudes and larger distances in order to eventually detect the length scale $r_{\mathrm{c}}$.

Up to now studies of the correlation function $\xi(r)$ in galaxy samples have been limited to small scales, i.e. $0.1<r \lessgtr$ 30 Mpc/h (i.e. Totsuji \& Kihara 1969; Davis \& Peebles 1983;

\footnotetext{
${ }^{1}$ For the sake of clarity, we choose the scale of distances normalized to the adimensional Hubble parameter $h$, which is defined from the Hubble constant $H_{0}=100 h \mathrm{~km} \mathrm{~s}^{-1} \mathrm{Mpc}^{-1}$.
}

Davis et al. 1988; Benoist et al. 1996; Park et al. 1994; Scranton et al. 2002; Zehavi et al. 2002, 2004; Ross et al. 2007), and only recently has the volume covered by galaxy redshift samples approached a size large enough to make a robust estimation of the correlation function on scales of about $100 \mathrm{Mpc} / \mathrm{h}$. When the Sloan Digital Sky Survey (SDSS) (York et al. 2000) is completed by filling up the gap between the two main angular regions of observations the volume of the survey and the statistics of the number of objects in the samples would be large enough to test space correlations on scales of order $r_{\mathrm{c}}$ or more. An exception to this situation is represented by the paper by Eisenstein et al. (2005), who, by studying a sample of luminous red galaxies (LRG) of the SDSS, have estimated the correlation function on scales around $100 \mathrm{Mpc} / \mathrm{h}$. These authors have focused their attention however on another real-space feature of theoretical models: the so-called "bump" of the correlation function, which corresponds in real space to the so-called Doppler peaks in the matter power spectrum generated by the baryonic acoustic oscillations in the early universe. As we discuss below, this bump, corresponding to a singular point of the correlation function (Gabrielli et al. 2004), is localized on scales close to $100 \mathrm{Mpc} / \mathrm{h}$ and characterized by a small amplitude. This is a second important real-space scale of the theoretical correlation function, which is localized on a scale slightly smaller than $r_{\mathrm{c}}$. The detection of the baryonic bump is thus related to the detection of the scale $r_{\mathrm{c}}$ as any finitesize effect perturbing the determination of the scale $r_{\mathrm{c}}$ will also inevitably affect the determination of the baryonic bump. In fact, the baryonic bump can be seen as a small modification of the overall shape of the correlation function on scales of about $r_{\mathrm{c}}$, on which we focus our attention here.

Note that, because of the very large scales, the acoustic signature and the zero point scale remain in the linear regime even today, and they are weakly affected by non-linear effects (see Eisenstein et al. 2006). Thus real-space and redshift-space properties on such large scales should not differ substantially.

In Sect. 2 we introduce the basic definitions of the statistical quantities usually employed to characterize two-point properties in real and Fourier space. In Sect. 3 we discuss a simple functional behavior of the power spectrum of matterdensity fluctuations, which captures the main elements of a more realistic CDM power-spectrum. We discuss the real-space properties as represented by the two-point correlation function and we consider the problem of selection or biasing in the simplest theoretical scheme of biasing a correlated Gaussian field. In Sect. 4 we explicitly treat the case of a $\Lambda$ CDM matter density field characterizing in detail real-space properties. The main estimators of the two-point correlation function are discussed in Sect. 5, and in Sect. 6 we test those estimators in artificial distributions. Finally in Sect. 7 we draw our main conclusions discussing the problems related to the estimations of two-point correlations in real galaxy samples.

\section{Basic definitions}

The microscopic number density function for any particle ${ }^{2}$ distribution is given by

$n(\boldsymbol{x})=\sum_{i=1}^{N} \delta_{\mathrm{D}}\left(\boldsymbol{x}-\boldsymbol{x}_{i}\right)$,

${ }^{2}$ We make it explicit that we consider particle distributions; however, most of the definitions given hereafter can be easily extended to the case of a continuous matter density field. We refer to Gabrielli et al. (2004) for more details. 
where $\boldsymbol{x}_{i}$ is the position of the $i$ th particle, $\delta_{\mathrm{D}}$ the Dirac delta function, and the sum is over the $N$ particles of the system.

For a system in which the mean density $n_{0}$ is well-defined and positive, it is convenient to define the density contrast:

$\delta(\boldsymbol{x})=\frac{n(\boldsymbol{x})-n_{0}}{n_{0}}$.

In order to characterize the two-point correlation properties of the density fluctuations, one can then use the reduced two-point correlation function (hereafter called simply the two-point correlation function):

$\tilde{\xi}(\boldsymbol{r})=\langle\delta(\boldsymbol{x}+\boldsymbol{r}) \delta(\boldsymbol{x})\rangle$,

where $\langle\ldots\rangle$ is the ensemble average, i.e., an average over all possible realizations of the system. In a distribution of discrete particles, $\tilde{\xi}(\boldsymbol{r})$ always has a Dirac delta function singularity at $\boldsymbol{r}=0$, which it is convenient to separate by defining $\xi(\boldsymbol{r})$ for $\boldsymbol{r} \neq 0$ (the "off-diagonal" part - see e.g. Peebles 1980):

$\tilde{\xi}(\boldsymbol{r})=\frac{1}{n_{0}} \delta_{\mathrm{D}}(\boldsymbol{r})+\xi(\boldsymbol{r})$.

The normalized variance of particle number (or mass) is an integrated quantity defined as

$\sigma^{2}(r)=\frac{\left\langle N^{2}(r)\right\rangle-\langle N(r)\rangle^{2}}{\langle N(r)\rangle^{2}}$

where $N(r)$ is the number of particles inside, for example, a sphere of radius $r$. Then $\sigma^{2}(r)$ can be used, in a manner similar to $\tilde{\xi}(\boldsymbol{r})$, to distinguish a regime of large fluctuations $\left(\sigma^{2}>1\right)$ from a regime of small fluctuations where $\sigma^{2}<1$. It is simple to find the explicit expression for the normalized variance of the particle number in terms of a double integral of $\tilde{\xi}(\boldsymbol{r})$ (see, e.g., Peebles 1980)

$\sigma^{2}(V)=\frac{1}{V^{2}} \int_{V} \int_{V} \tilde{\xi}\left(\left|\boldsymbol{r}_{1}-\boldsymbol{r}_{2}\right|\right) \mathrm{d}^{3} r_{1} \mathrm{~d}^{3} r_{2}$.

If we consider distributions that are periodic in a cube of side $L$, we can write the density contrast as a Fourier series:

$\delta(\boldsymbol{x})=\frac{1}{L^{3}} \sum_{k} \exp (\mathrm{i} \boldsymbol{k} \cdot \boldsymbol{x}) \tilde{\delta}(\boldsymbol{k})$

with $k \in\left\{(2 \pi / L) \boldsymbol{n} \mid \boldsymbol{n} \in Z^{3}\right\}$. The coefficients $\tilde{\delta}(\boldsymbol{k})$ are given by

$\tilde{\delta}(\boldsymbol{k})=\int_{L^{3}} \delta(\boldsymbol{x}) \exp (-\mathrm{i} \boldsymbol{k} \cdot \boldsymbol{x}) \mathrm{d}^{3} \boldsymbol{x}$.

The power-spectrum of a particle distribution is then defined (see e.g., Peebles 1980) as

$P(\boldsymbol{k})=\frac{1}{L^{3}}\left\langle|\tilde{\delta}(\boldsymbol{k})|^{2}\right\rangle$.

In point distributions which are statistically homogeneous, the power spectrum and the non-diagonal part of the two-point correlation function $\xi(\boldsymbol{r})$ are a Fourier conjugate pair:

$\xi(\boldsymbol{r})=\frac{1}{(2 \pi)^{3}} \int \mathrm{d}^{3} k P(\boldsymbol{k}) \exp (-\mathrm{i} \boldsymbol{k} \boldsymbol{r})$

and

$P(\boldsymbol{k})=\int \mathrm{d}^{3} r \xi(\boldsymbol{r}) \exp (\mathrm{i} \boldsymbol{k r})$.

Since for both $\xi(\boldsymbol{r})$ and $P(\boldsymbol{k})$ we consider only the dependence on the modulus of their arguments, we will denote them from now on as $\xi(r)$ and $P(k)$ to mean that they are obtained by performing an average over the directions of $\boldsymbol{r}$ and $\boldsymbol{k}$, respectively.

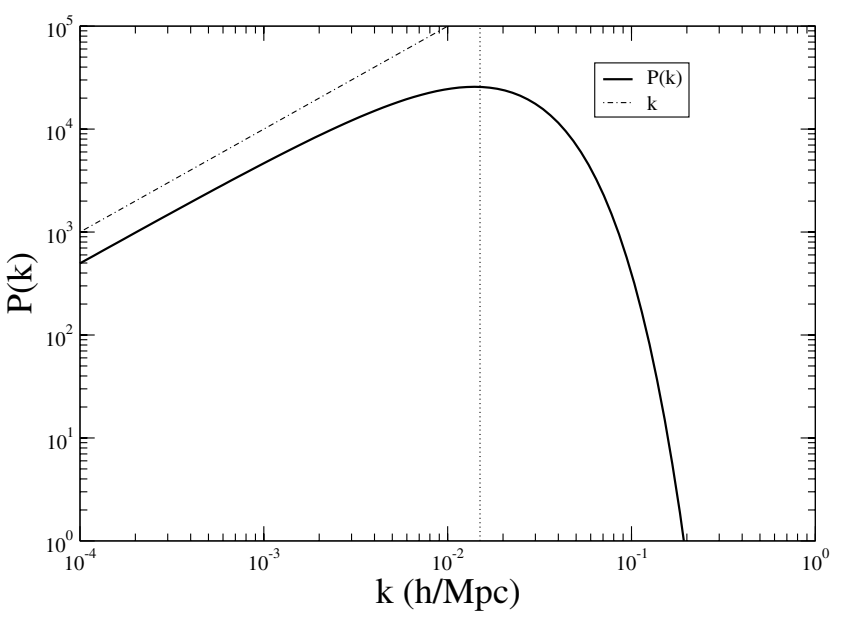

Fig. 1. Power spectrum given by Eq. (12). The linear behavior at small $k$ is reported as a reference. The amplitude at small $k$ and the scale $k_{\mathrm{c}}=$ $0.014 \mathrm{~h} / \mathrm{Mpc}$ are chosen to be the same of the $\Lambda \mathrm{CDM}$ models discussed in what follows. The vertical lines indicates the wavelength $k_{\mathrm{c}}$.

\section{A toy model and the problem of sampling}

To illustrate some key features of standard cosmological models, let us consider a simple matter-density field power spectrum of the type:

$P(k)=A k \exp \left(-k / k_{\mathrm{c}}\right)$.

This is characterized by an amplitude $A$ that fixes the small $k$ behavior and by the turnover scale $k_{\mathrm{c}}$ (see Fig. 1).

As already mentioned, the two-point correlation function is simply the Fourier transformation (FT) of the power spectrum: for Eq. (12) by using Eq. (6) we find

$\xi(r)=\frac{A}{\pi^{2}} \frac{\left(\frac{3}{k_{\mathrm{c}}^{2}}-r^{2}\right)}{\left(\frac{1}{k_{\mathrm{c}}^{2}}+r^{2}\right)^{3}}$.

This correlation function presents the zero point at the intrinsic characteristic scale

$r_{\mathrm{c}}=\sqrt{3} / k_{\mathrm{c}}$.

On small scales $r \ll r_{\mathrm{c}}$ Eq. (13) gives $\xi(r) \approx$ const. $>0$; while at large scales $r \gg r_{\mathrm{c}}$ the amplitude of $\xi(r)$ becomes negative, going to zero for $r \rightarrow \infty$ with a power-law tail of the type $\xi(r) \approx$ $-r^{-4}$ (see Fig. 2).

The region of positive correlation is thus followed by an (infinite) region where there are anti-correlations. Positive and negative correlations are exactly balanced so that

$\int_{0}^{\infty} \xi(r) r^{2} \mathrm{~d} r=0$

This is equivalent to the condition that $P(k) \rightarrow 0$ for $k \rightarrow 0$. As discussed in Gabrielli et al. (2002) (see also Gabrielli et al. 2004), this corresponds to the fact that the distribution is globally super-homogeneous, i.e. more ordered than an uncorrelated distribution (i.e. a Poisson). This subtle property can be clarified by computing the mass variance.

To evaluate the mass variance (Eq. (6)), one may choose a sphere in real space of radius $R$ as the volume of integration $V$. 


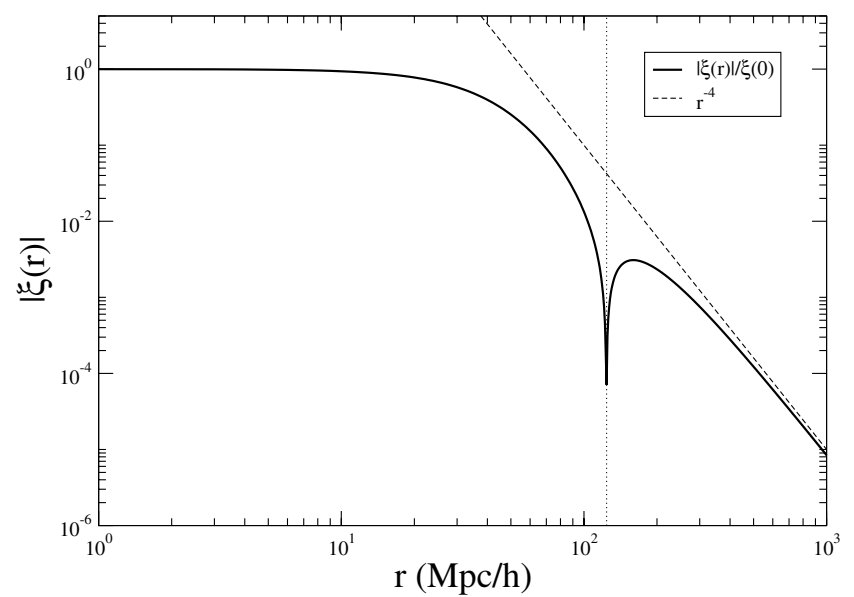

Fig. 2. Absolute value of the two-point correlation function given by Eq. (13) divided by $\xi(0)$. The (negative) power-law $r^{-4}$ is shown as a reference. The vertical line indicates the scale $r_{\mathrm{c}}$.

In this case, going into Fourier space, Eq. (6) becomes (see e.g., Peebles 1980)

$\sigma^{2}(R)=\frac{9}{2 \pi^{2}} \int_{0}^{\infty} \mathrm{d} k k^{2} P(k) \frac{(\sin (k R)+(k R) \cos (k R))^{2}}{(k R)^{6}}$.

By considering the power spectrum given by Eq. (12), one finds that $\sigma^{2}(R) \approx$ const. for $R<r_{\mathrm{c}}$ and $\sigma^{2}(R) \sim R^{-4}$ for $R>r_{\mathrm{c}}$ (see Fig. 3). This fast decay of the mass variance is the distinctive feature of super-homogeneous mass distributions, and it is strictly related to the condition $P(0)=0$. This is the fastest decay possible for any isotropic translationally invariant distribution of points (see discussion in Gabrielli et al. 2002).

For a Poisson distribution one finds that the mass variance decay more slowly than for a super-homogeneous distribution, i.e. $\sigma^{2}(R) \sim R^{-3}$, and that the power spectrum obeys

$\lim _{k \rightarrow 0} P(k)=$ const. $>0$.

A similar situation occurs when the distribution has positive correlations on small scales and no correlations at large scales a substantially Poisson distribution. On the other hand, in the presence of long-range positive correlations, as for example a power-law correlation function $\xi(r) \sim r^{-\gamma}$, with $0<\gamma<3$, the mass variance decays more slowly than the Poisson case, i.e. $\sigma^{2}(R) \sim R^{(\gamma-3)}$, and the power spectrum satisfies the condition

$\lim _{k \rightarrow 0} P(k)=\infty$.

\subsection{Sampling a density field}

What we have just described is a simple toy model power spectrum that captures some essential features of the theoretical correlation properties of the matter density field. In the discussion of galaxy samples, one has to consider that luminous objects trace the underlying dark-matter density field and that they can be regarded as a sampling of it: for example they can be supposed to lie in the highest peaks of the fluctuations field, because only there has gravitational clustering been efficient enough to form self-gravitating objects. The problem of sampling is thus a central one in studies of cosmological density fields and, particularly, of galaxy structures. More precisely we mean by sampling the operation performed when one extracts, from a given distribution, a sub-sample of it by using a selection criteria based on

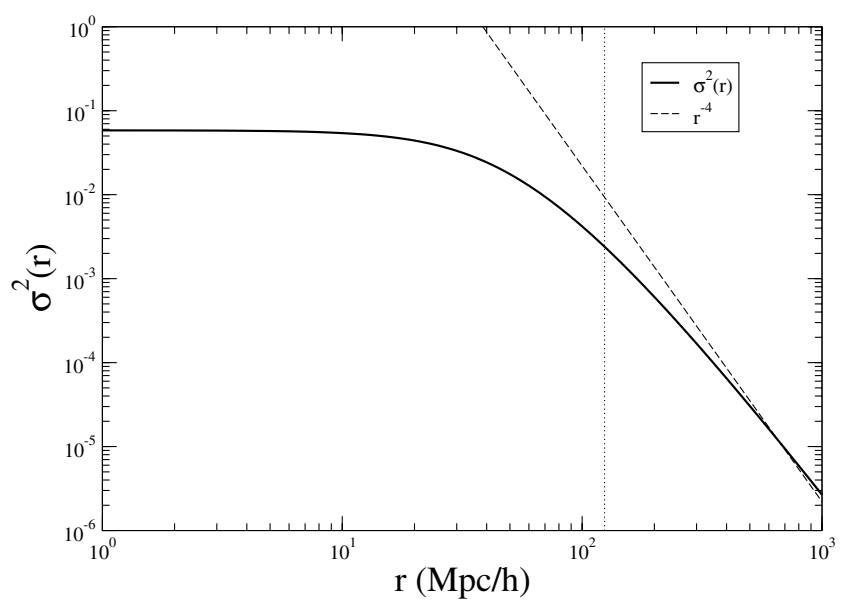

Fig. 3. Variance in real-space spheres for Eq. (12). A line with slope $r^{-4}$ is reported as reference. The vertical line indicates the scale $r_{\mathrm{c}}$.

a certain parameter characterizing the distribution. For example, one can make this type of selection by extracting only those objects whose luminosity is brighter than a given threshold from the whole population of galaxies of all luminosity. Alternatively, a similar selection can be done by considering galaxy color. In the case the fluctuation field is a stochastic variable of position (for example a Gaussian fluctuation field), one may sample the distribution by selecting only those fluctuations larger than a given threshold in the density fluctuation field.

In general the problem consists of the understanding of the relations between the statistical properties of the sampled, or biased, distribution with those of the original one. A particular interest lies in the relation between the two-point correlation function of the sampled field with the original $\xi(r)$. This is because, for instance, in the studies of galaxy samples, one naturally has to perform a sampling when measuring the two-point correlation function of galaxies of a certain luminosity. In the comparison of observations with theoretical models, the sampling procedure is strictly related to the physics of the system. In fact, in the analysis of cosmological $N$-body simulations, one also needs to extract sub-samples of points that, according to some models, would represent galaxies instead of dark-matter particles. In these contexts, the simplest theoretical model describing biasing (introduced by Kaiser 1984) was developed for a continuous Gaussian field, and thus it does not represent a useful analytical treatment of the problem of strong clustering, which is instead the relevant one for galaxy structures.

However it is very difficult to treat the problem of sampling for a generic case unless one may specify the correlation properties in detail of the original distribution and the specific procedure used to make the sampling. This is a task beyond current knowledge even for the case of artificial distributions generated by gravitational $N$-body simulations where one can take a phenomenological approach. For this reason, we limit the discussion to the threshold sampling of the Gaussian random fields, because this allows us to point out some key-features characterizing the case in which the underlying density field has superhomogeneous type correlations and the sampling is local (i.e. related to local features of the distribution). This cannot be regarded as a realistic example for the reasons discussed above, but one may identify several key problems that should be addressed in detail by means of studies of artificial distributions generated, for example, by $\mathrm{N}$-body simulations for the understanding of a more realistic case. 


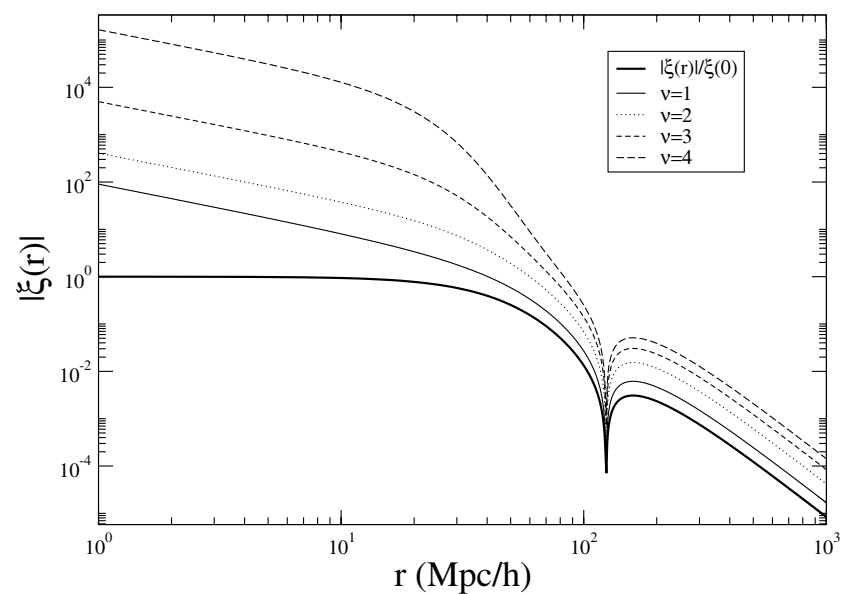

Fig. 4. Absolute value of the correlation function of the toy model described by Eq. (12) (solid line) and of the ones corresponding to different values of the threshold parameter $v$ calculated by applying Eq. (20). The amplification is non-linear on small scales, where $\xi_{\tilde{\mu}}(r)>1$, linear on large scales, and the zero-crossing scale is invariant under biasing.

\subsection{Sampling a Gaussian random field}

Let us now discuss the simplest biasing scheme of a continuous and correlated Gaussian field (hereafter we follow Durrer et al. 2003). Suppose having a Gaussian random field with two-point correlation $\xi(r)$ such that the variance is $\left\langle\mu^{2}\right\rangle=\sigma^{2}$ (where $\mu$ is the mean density normalized fluctuation). One can identify fluctuations in the field such that they are larger than $v$ times the variance. This selection defines a biased field with the weight equal to zero if the fluctuations of the original field are smaller than $\tilde{\mu} \equiv \nu \sigma$ and equal to one if they are equal to, or larger than $\tilde{\mu}$. When one changes the threshold $v$ one selects different regions of the underlying Gaussian random field, corresponding to fluctuations of differing amplitudes. The two-point correlation function of the selected objects is then that of the peaks $\xi_{\tilde{\mu}}(r)$.

We define the two-point correlation function of the normalized field

$\hat{\xi}(r)=\frac{\xi(r)}{\xi(0)}$,

where $\xi(0)$ is the variance of the field so that $\hat{\xi}(r) \leq 1 \forall r$. It is possible to compute the following first-order approximation (Durrer et al. 2003)

$\xi_{\tilde{\mu}}(r) \approx \sqrt{\frac{1+\hat{\xi}(r)}{1-\hat{\xi}(r)}} \exp \left(v^{2} \frac{\hat{\xi}(r)}{1+\hat{\xi}(r)}\right)-1$,

which reduces to $\xi_{\tilde{\mu}}(r) \simeq v^{2} \hat{\xi}(r)$ when $v^{2}|\xi(r)| \ll 1$. Thus, if present in the underlying distribution, the characteristic length scale of the zero point $r_{\mathrm{c}}$ is not changed under this selection procedure, i.e.,

$\xi_{\tilde{\mu}}\left(r_{\mathrm{c}}\right)=\hat{\xi}\left(r_{\mathrm{c}}\right)=0 \quad \forall \tilde{\mu}$.

On the other hand, for $\xi_{\tilde{\mu}}(r)>1$ the amplification is non-linear as a function of scale: this means that the functional behavior of $\xi_{\tilde{\mu}}(r)$ is different from the one of $\hat{\xi}(r)$ in the regime where $\xi_{\tilde{\mu}}(r)>1$. Figure 4 shows the situation when one takes the correlation function of the toy model discussed in the previous section (see Eq. (13)) as the $\xi(r)$ of the underlying Gaussian field.

Given the asymmetrical amplification on small and on large scales the condition of super-homogeneity is broken, i.e.

$\int_{0}^{\infty} \xi_{\tilde{\mu}}(r) r^{2} \mathrm{~d} r>0$

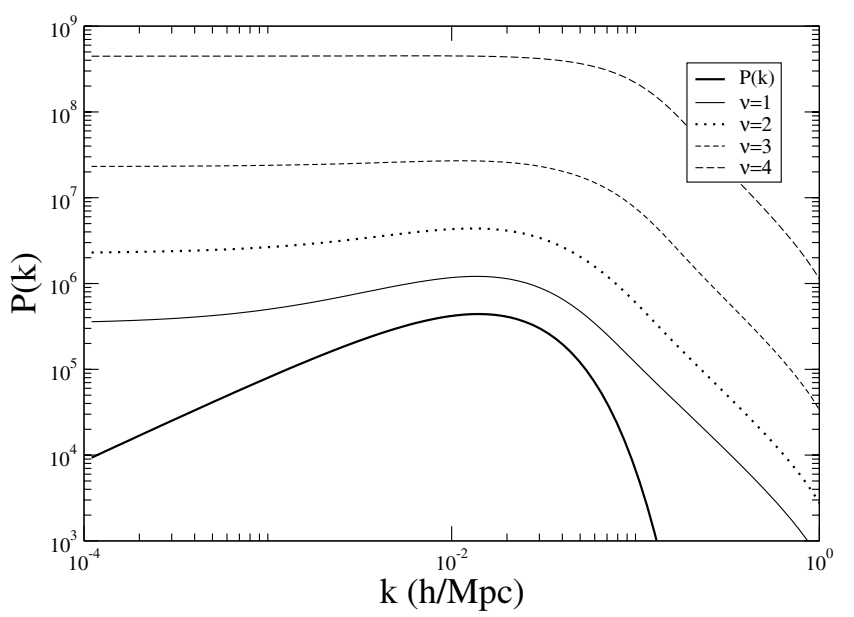

Fig. 5. Power spectrum of the toy model described by Eq. (12) (solid line) and of the ones corresponding to different values of the threshold parameter $v$ calculated by applying Eq. (20) and then by making the Fourier transformation. Because of the asymmetrical amplification of the correlation function on small and on large scales the condition of super-homogeneity is broken, i.e. the power spectrum does not show anymore the tail $P(k) \sim k$.

and thus the power spectrum no longer show the tail $P(k) \sim$ $k$ (see Fig. 5). Correspondingly the mass variance shows the typical features of a substantially Poisson system beyond the scale $r_{\mathrm{c}}$, i.e. it decays as $r^{-3}$.

Summarizing the behaviors for the toy model described by Eq. (12), we find that:

- (i) the correlation function of the biased field still presents some key features of the original correlation function, namely the same characteristic scale $r_{\mathrm{c}}$ and the same negative tail $\xi(r) \sim-r^{-4}$ on large scales;

- (ii) the power spectrum is distorted in a non-linear way on all scales by biasing; in particular on large scales this is characterized by the typical behavior of a Poisson distribution. The same situation occurs for the mass variance.

We expect these to be general features of the biased fields when the underlying density field has super-homogeneous type correlations (Durrer et al. 2003; Gabrielli et al. 2004). The cancellation of the super-homogeneous features stems for the operation of selection introducing a noise, due to the sampling itself, which dominates the intrinsic fluctuations of the system.

\section{Real space correlations in CDM-type models}

In this section we consider the case of a distribution with correlation properties of CDM type. In particular we study the case of the so-called $\Lambda C D M$ "vanilla" model. The functional behavior and the parameters defining this model are discussed in Tegmark et al. (2004) and Spergel et al. (2007). Without entering into the details of the model here, we note that, while the different cosmological parameters may change the behavior of the power spectrum in a non-linear way, it is generally assumed that the bias factor $b$ (we now indicate by $b$ what in the previous section we called $v$ to make clear that the latter symbol refers only to the case of a correlated Gaussian field) corresponds to an overall rescaling of its amplitude:

$P(k)=b^{2} P_{\mathrm{dm}}(k)$, 


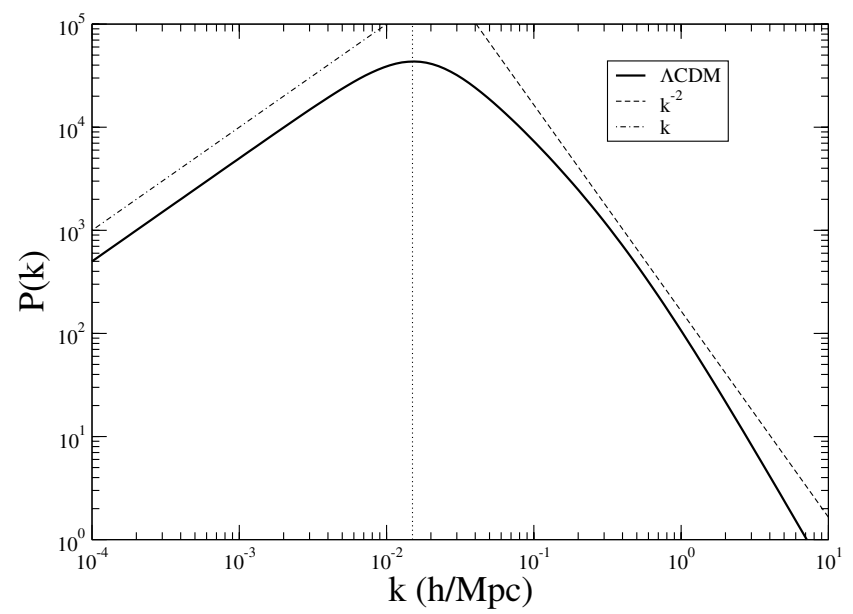

Fig. 6. Power spectrum for the $\Lambda$ CDM model (Eq. (24)). The two power laws $P(k) \sim k$ and $P(k) \sim k^{-2}$ are shown as a reference.

where $P_{\mathrm{dm}}(k)$ represents the power spectrum of the underlying dark-matter field and $P(k)$ is the "biased" power spectrum, corresponding to the power spectrum of a field selected by following a certain prescription. As discussed above, Eq. (23) does not have any theoretical justification in the framework of Gaussian fields, whether at small $k$ or at large $k$. In numerical simulations it has instead been phenomenologically found that this is a good working hypothesis in the regime of strong clustering (Springel et al. 2005).

To compute the real-space properties, it is useful to find an analytical approximation to the theoretical power spectrum that can be found numerically (we use hereafter the data from Tegmark et al. 2004). We have found that the following expression provides us with a good fitting formula

$$
P(k)=\frac{A k}{\left(1+B\left(k / k_{1}\right)^{v_{1}}+\left(k / k_{2}\right)^{v_{2}}\right)}
$$

where $A=5 \times 10^{6}, B=10^{3}, k_{1}=0.35 \mathrm{~h} / \mathrm{Mpc}, v_{1}=2.3, k_{2}=$ $0.05 \mathrm{~h} / \mathrm{Mpc}, v_{2}=3.5$. This power spectrum is characterized by a turnover scale $k_{\mathrm{c}} \approx 0.014 \mathrm{~h} / \mathrm{Mpc}$, which separates the large-scale behavior $P(k) \sim k$ from the small-scale one $P(k) \sim k^{-2}$.

In this case it is not possible to calculate the real-space correlation function analytically, but it can be obtained from the numerical computation of the Fourier transform of the power spectrum by using Eq. (10). The result is shown in Fig. 7. As for the case of the toy model discussed in the previous section, this correlation function is characterized by a positive region at small scales, where in this case it decays roughly as $r^{-1.5}$, and by a large scale negative tail $\xi(r) \sim-r^{-4}$. The length scale which separates these two regimes is the zero-point $r_{\mathrm{c}}$ which represents the unique characteristic length scale of this model: for the parameters chosen in Eq. (24) we find $r_{\mathrm{c}}=124 \mathrm{Mpc} / \mathrm{h}$.

A reasonable fit to the correlation function obtained by making the FT is (see Fig. 8)

$\xi(r)=\frac{A}{\pi^{2}} \frac{\left(\frac{3}{k_{\mathrm{c}}^{2}}-r^{2}\right)}{\left(\frac{1}{k_{\mathrm{c}}^{2}}+r^{2}\right)^{3}} \cdot\left(\frac{r^{\beta}+\frac{3^{(\beta-2) / 2}}{k_{\mathrm{c}}^{\beta}}}{r^{\beta}}\right)$

where $A=5 \times 10^{6}$ and $k_{\mathrm{c}}=0.014 \mathrm{~h} / \mathrm{Mpc}$ and $\beta=1.4$.

It is interesting to note that if we compute the power spectrum by calculating the FT of the correlation function by using the analytical approximation given by Eq. (25), although the fit

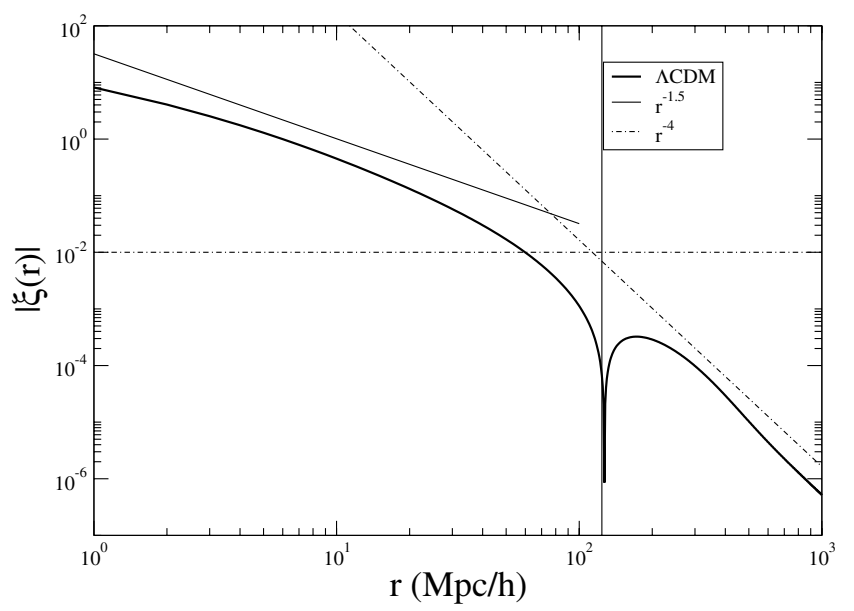

Fig. 7. Absolute value of the two-point correlation function for the $\Lambda \mathrm{CDM}$ model (Eq. (24)). The two power laws $r^{-1.5}$ and $r^{-4}$ are shown as a reference.

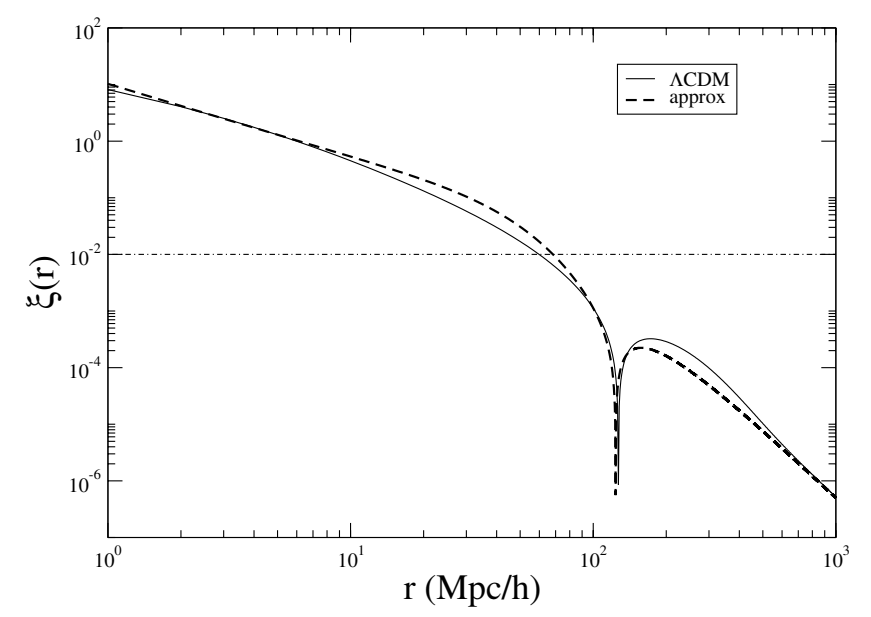

Fig. 8. Correlation function for the $\Lambda \mathrm{CDM}$ model (Eq. (24)) and the approximation given by Eq. (25).

is very good over the all range of scales considered, we do not get the correct behavior at small wave modes, i.e. that $P(k) \sim k$ for $k<k_{\mathrm{c}}$. Instead we get $P(k) \sim$ const. for $k<k_{\mathrm{c}}$ (see Fig. 9). This is because the small approximation introduced in Eq. (25) is such that the integral

$$
\int_{0}^{\infty} \xi(r) r^{2} \mathrm{~d} r>0
$$

and thus there is no the perfect cancellation between the positive and negative parts, i.e. the typical feature of super-homogeneous distributions, characterized by an extremely fine-tuning of the correlations. This simple example shows how reasonable the condition of super-homogeneity is and gives a feeling of the kind of problems that can arise in the framework of sampling. In general, the amplification of the correlation function due to selection (or bias) is not linear and gives rise to a behavior like one just described, i.e. to the radical change of the superhomogeneous properties. That is, the distribution becomes substantially Poisson on scales larger than $r_{\mathrm{c}}$ because of the noise introduced by sampling, although the negative $-r^{-4}$ tail in the correlation function is still present. 


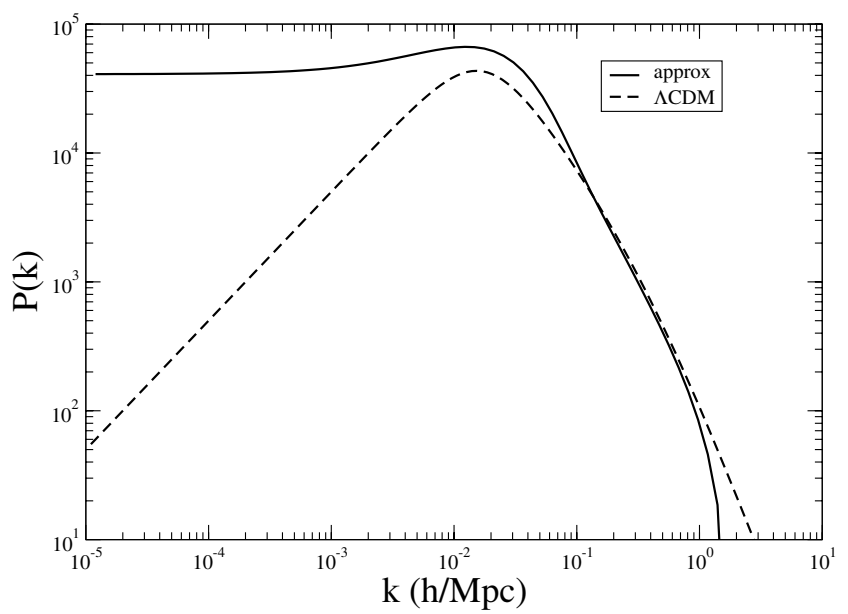

Fig. 9. Power spectrum for the $\Lambda \mathrm{CDM}$ model and the approximation obtained from Eq. (11) by using Eq. (25).

\subsection{Main features of the real-space two-point correlation function}

As discussed above, the regime of large fluctuations $\xi(r)>1$ is not predictable by a theoretical approach, and thus both the amplitude and the shape of the correlation function have to be constrained by observations. Any specific model of matter density field, however, predicts the behavior of the correlation function in the regime $|\xi(r)|<1$. We discuss, as an interesting example, the case of the $\Lambda$ CDM model mentioned above.

In general, it is possible to characterize the approach of the correlation function to the zero point, in a range of scales such that $0<\xi(r)$. For the case of the $\Lambda$ CDM model, we find that, in this range of scales, a good and useful approximation is given by

$\xi(r) \approx A\left(\frac{\lambda}{r}\right)^{\gamma} \exp (-r / \lambda)$

where $A=3 \times 10^{-1}, \lambda=25 \mathrm{Mpc} / \mathrm{h}$, and $\gamma=1$, while $A=3(0.03)$ when the amplitude of Eq. (24) is multiplied by a factor $10(1 / 10)$. The result is shown in Fig. 10. The exponential cut-off, independent of bias, is related to the fact that $\xi(r)$ crosses zero at $r_{\mathrm{c}}=124 \mathrm{Mpc} / \mathrm{h}$. Thus, while the direct identification of the zero-point scale is clearly very difficult in a finite sample (see discussion below), for the effect of stochastic and systematic noise in the estimators, the approach to the zero point, in this model, is very well defined. In particular, the correlation function presents an exponential decay in the range of scales $[10,100] \mathrm{Mpc} / \mathrm{h}$. Depending on the value of the amplitude of $\xi(r)$, this range of scales is extended enough in the region where $\xi(r)>a$ with $a>10^{-2}$, thus a region where maybe observations will be provide with statistically robust samples, for a bias factor of order one for the parameters considered here.

\subsection{The baryonic bump}

As mentioned in the introduction, according to the physics of the early universe, sound waves propagating in the first $\sim 400000$ years after the Big Bang produce an additional characteristic length scale in the matter and radiation density fields. With galaxy surveys, it would be possible to detect this acoustic feature as a bump in the correlation function at $\sim 100 \mathrm{Mpc} / \mathrm{h}$. The amplitude of this bump is controlled by the baryon density, the matter density, and the Hubble constant (see Eisenstein et al. 2005 , for a detailed discussion). It is interesting to note that this

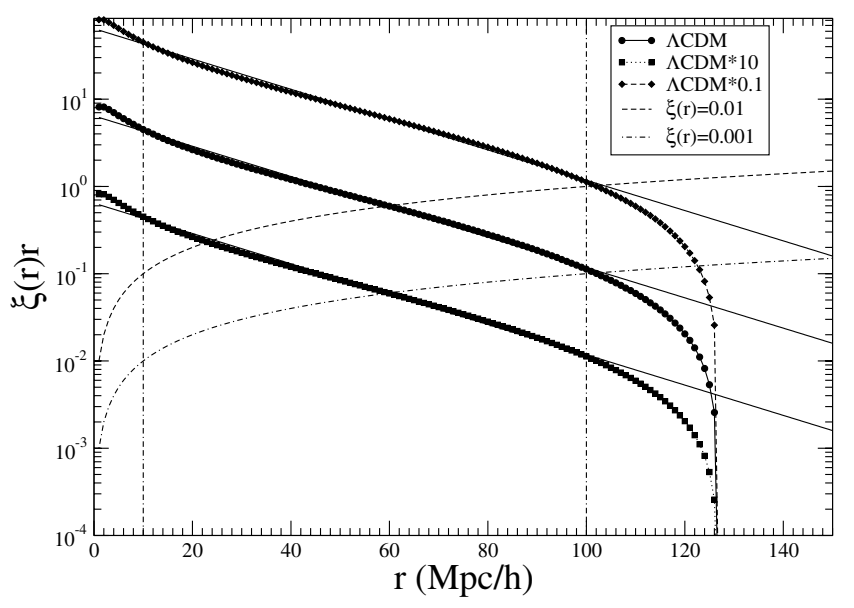

Fig. 10. Plot of the function $\xi(r) \times r$ for the $\Lambda$ CDM model (Eq. (24)) and, for comparison for the case in which the amplitude of Eq. (24) as been multiplied by a factor 10 and a factor $1 / 10$. The dashed lines correspond to the thresholds such that $\xi(r)=0.01,0.001$.

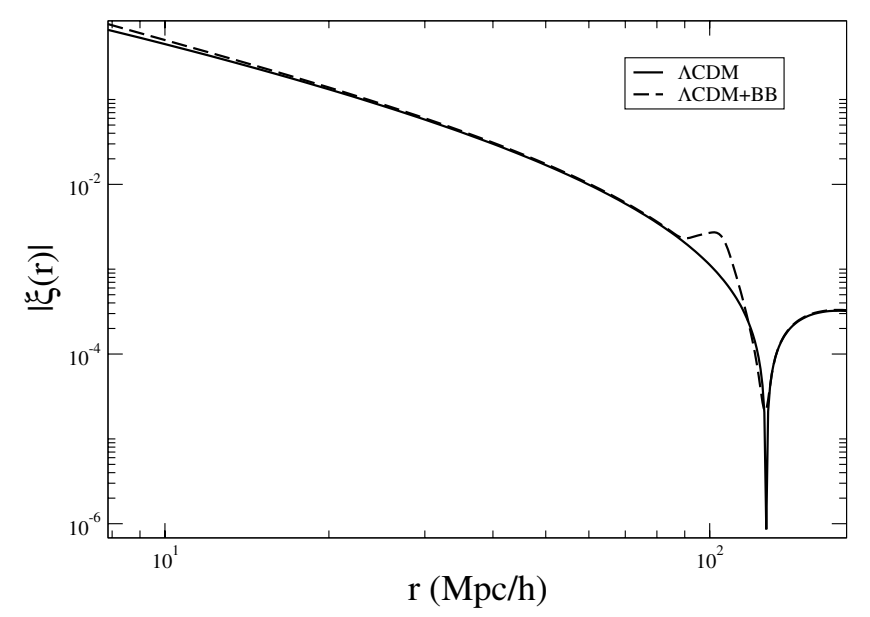

Fig. 11. Absolute value of the two-point correlation function for the $\Lambda \mathrm{CDM}$ model (Eq. (24)) and for the same model with the baryonic bump (BB) at $\sim 100 \mathrm{Mpc} / \mathrm{h}$.

bump corresponds to a non-analytical point of the correlation function, which gives rise to a co-sinusoidal modulation for the power spectrum (see Gabrielli et al. 2004).

In Fig. 11 we show a typical example. (In this the case the matter density is $\Omega_{\mathrm{m}}=0.12 h^{-2}$ and the baryon density is $\Omega_{\mathrm{b}}=$ $0.024 h^{-2}$.) As one may notice from this figure, the bump appears as a very small amplitude feature of the two-point correlation function localized at about $\sim 100 \mathrm{Mpc} / \mathrm{h}$, i.e. when the correlation function shows the sharp break corresponding to the approach to the zero point, which fixes the global shape of the correlation function on those scales. As we discuss below, one of the main problems in the estimation of the correlation function on such scales in a given finite sample is to establish whether the break in the power-law behavior, i.e., the overall shape corresponding to the presence of the zero-point scale, is biased or not by a finite size effect. Once certain enough that the shape is not affected by systematic effects, then one may try to characterize the presence of the baryonic feature. 


\section{Estimation of the correlation function}

Different estimators of the two-point correlation function have been introduced and discussed in the literature. The difference between them lies in their respective methods of edge corrections (Kerscher et al. 2000), which gives rise to different variance and systematic effects or biases. We discuss three of them: (i) the full-shell (FS) estimator (Gabrielli et al. 2004), (ii) the Landy and Szalay (LS) estimator (Landy \& Szalay 1993), and (iii) the Davis and Peebles (DP) estimator (Davis \& Peebles 1983). The first one has the advantage that all biases can be carefully understood and possibly kept in control. The second is very popular because it has the minimal variance for the case of a Poisson distribution, although it has not been demonstrated that the same minimal variance applies in case of correlated distributions (see e.g. Kerscher et al. 2000). However, it has the disadvantage that the biases are very poorly understood in the general case, as in the case of the DP estimator. Although there have been several studies of these estimators (see e.g. Kerscher 1999; and Kerscher et al. 2000), systematic tests for biases are still not completely developed. Here we introduce the problem and analyze the case of the FS estimator, while in the next section we try to quantify the problem by studying numerical simulations.

Note that there are, at least, three other estimators known in the literature: the natural estimator, the Hewett estimator and the Hamilton estimator, generally all biased as the LS and DP estimators. In a detailed comparison between these estimators performed by Kerscher et al. (2000) it is reported that the performance of the LS estimator is almost indistinguishable from the Hamilton estimator. In addition, after a careful study, Kerscher et al. (2000) have stressed that the LS estimator is the recommended one. For this reason we decided to focus our studies on the LS, while we choose the DP because it is commonly used in the literature.

\subsection{Bias in the estimators}

Let us call $\overline{X(V)}$ the statistical estimator of an average quantity $\langle X\rangle$ in a volume $V$ (where $\langle X\rangle$ denotes the ensemble average and $\bar{X}$ the sample average). To be a valid estimator $\overline{X(V)}$ must satisfy (Gabrielli et al. 2004)

$\lim _{V \rightarrow \infty} \overline{X(V)}=\langle X\rangle$.

A stronger condition is that the ensemble average of the estimator, in a finite volume $V$, is equal to the ensemble average $\langle X\rangle$ :

$\langle\overline{X(V)}\rangle=\langle X\rangle$.

An estimator is called unbiased if this condition is satisfied; otherwise, there is a systematic bias in the finite volume relative to the ensemble average. Any estimator $\overline{\xi(r)}$ of the correlation function $\xi(r)$, is generally biased. This is because the estimation of the sample mean density is biased when correlations extend over the sample size and beyond. In fact, the most common estimator of the average density is

$\bar{n}=\frac{N}{V}$,

where $N$ is the number of points in a sample of volume $V$. It is simple to show that (see, e.g., Gabrielli et al. 2004)

$\langle\bar{n}\rangle=\langle n\rangle\left(1+\frac{1}{V} \int_{V} \mathrm{~d}^{3} r \xi(r)\right)$.
Therefore only in case when $\xi(r)=0$ (i.e. for a Poisson distribution), Eq. (30) is an unbiased estimator of the ensemble average density.

In Kerscher (1999) one may find a detailed treatment of estimators of the two-point correlation function. It is shown that in a given sample, on large scales, the biases in the above-mentioned estimators are not negligible especially when there are structures of large spatial extension inside a given sample. In $\Lambda \mathrm{CDM}$ models, there are structures of large amplitude at small scales, i.e. up to $\sim 10 \mathrm{Mpc} / \mathrm{h}$, and structures of large spatial extension and low amplitude up to $\sim 120 \mathrm{Mpc} / \mathrm{h}$. Beyond such a scale there will be no structures anymore, as the distribution becomes anticorrelated. Thus it is important to understand the problem of biases in relation to real sample estimations, which may cover a distance scale of only several hundred $\mathrm{Mpc} / \mathrm{h}$, i.e. up to about five times the regime of positive correlations.

An analytical treatment of the problem, for the general case, is unfeasible, so that the most direct way to study biases in the estimators is by performing tests on artificial distributions, which we discuss in the next section. In what follows we present several examples that show the importance of the systematic effect related to Eq. (31), i.e. that the estimators do not satisfy, in general, Eq. (29) but only Eq. (28).

\subsection{The full shell estimator}

The correlation function can be written as

$\xi(r) \equiv \frac{\langle n(r) n(0)\rangle}{n_{0}^{2}}-1 \equiv \frac{\langle n(r)\rangle_{\mathrm{p}}}{n_{0}}-1$,

where the conditional density $\langle n(r)\rangle_{\mathrm{p}}=\langle n(r) n(0)\rangle / n_{0}$ gives the average number of points in a shell of radius $r$ and thickness $\mathrm{d} r$ from an occupied point of the distribution. Thus FS estimator (Gabrielli et al. 2004) can be simply written as

$\overline{\xi(r)}=\frac{\overline{(n(r))_{\mathrm{p}}}}{\bar{n}}-1$

where $\bar{n}$ is the estimated number density in the sample and $\overline{(n(r))_{\mathrm{p}}}$ is the estimator of the conditional density. The latter can be written as

$\overline{(n(r))_{\mathrm{p}}}=\frac{1}{N_{\mathrm{c}}(r)} \sum_{i=1}^{N_{\mathrm{c}}(r)} \frac{\Delta N_{i}(r, \Delta r)}{\Delta V}$,

where $\Delta N_{i}(r, \Delta r)$ is the number of points in the shell of radius $r$, thickness $\Delta r$, and volume $\Delta V=4 \pi r^{2} \Delta r$ centered on the $i$ th point of the distribution. Note that the number of points $N_{\mathrm{c}}(r)$ contributing to the average in Eq. (34) is scale-dependent, as only those points are considered such that when chosen as a center of the sphere of radius $r$, this is fully included in the sample volume (see Gabrielli et al. 2004; Vasilyev et al. 2006, for more details).

The sample density can be estimated in various ways. Suppose that the sample geometry is simply a sphere of radius $R_{\mathrm{s}}$. The most convenient in this context is to choose

$\bar{n}=\frac{3}{4 \pi R_{\mathrm{s}}^{3}} \int_{0}^{R_{\mathrm{s}}} \overline{(n(r))_{\mathrm{p}}} 4 \pi r^{2} \mathrm{~d} r$

as in this case the following integral constraint is satisfied

$\int_{0}^{R_{\mathrm{s}}} \overline{\xi(r)} r^{2} \mathrm{~d} r=0$ 
This condition is satisfied independently of the functional shape of the underlying correlation function $\xi(r)$.

The scale $R_{\mathrm{s}}$, for a sample of arbitrary geometry, is given by the radius of the maximum sphere fully contained in the sample volume for the reasons explained above. Other choices for estimating the sample density are possible and give rise to a condition of the type Eq. (36), even if not precisely the same. This condition introduces a systematic distortion in the measured shape of $\overline{\xi(r)}$, so the advantage in choosing Eq. (35) lies in the good control on the scale $r_{*}$, defined to be the scale beyond which the distortion becomes important. The scale $r_{*}$ must be evaluated given a specific model for $\xi(r)$, but it is generally a fraction of $R_{\mathrm{s}}$.

Thus the integral constraint for the FS estimator, Eq. (36), does not simply introduce an offset, but also a change in the functional behavior of the estimated correlation function. Other choices introduce distortions on a scale that is difficult to evaluate, especially in the case where the sample does not have a simple spherical geometry. In general, any estimator is distorted at some scales by a condition of the type given by Eq. (36), which basically reflects our ignorance of the value of the ensemble average density.

To study the effect of the integral constraint on the FS estimator, let us rewrite the estimation of the correlation in terms of the theoretical correlation function

$\overline{\xi(r)}=\frac{1+\xi(r)}{1+\frac{3}{R_{\mathrm{s}}^{3}} \int_{0}^{R_{\mathrm{s}}} \xi(r) r^{2} \mathrm{~d} r}-1$.

By writing Eq. (37), we assume that the stochastic noise is negligible, which of course is not a good approximation on any scale. However in this way we may be able to understand the effect of the integral constraint for the FS estimator. From Eq. (37) it is clear that this estimator is biased, as it does not satisfy Eq. (29) but only Eq. (28).

Let us consider two useful examples of the theoretical correlation function (i) $\xi(r) \sim r^{-\gamma}$ and in (ii) the $\Lambda$ CDM model of Eq. (25). The distortion due to the integral constraint in the FS estimator when the theoretical correlation function shows a power-law behavior with exponent $\gamma=2$ is illustrated in Fig. 12. One may see that, at $r \approx R_{\mathrm{s}} / 3$ the estimation is already distorted and, when $r \approx R_{\mathrm{S}} / 2$, the function $\overline{\xi(r)}$ crosses zero and becomes negative in order to satisfy Eq. (36).

The case of the $\Lambda C D M$ model is shown in Fig. 13. The situation is similar to the power-law case as long as one considers $R_{\mathrm{S}}$ smaller than the zero-point scale $r_{\mathrm{c}}$. For larger $R_{\mathrm{s}}$, one may see that zero point is not changed anymore, while the negative tail continues to be amplified in a non-linear way even on scales $r<R_{\mathrm{s}}$. For example, with a sample of size $R_{\mathrm{s}} \approx 600 \mathrm{Mpc} / \mathrm{h}$, the distortion of the power-law tail does not allow detection the $\xi(r) \sim-r^{-4}$ behavior, which is marginally visible only when $R_{\mathrm{s}}>1000 \mathrm{Mpc} / \mathrm{h}$.

\subsection{Pairwise estimators}

To determine a pairwise estimator, we define the following quantities. The number of data-data pairs

$D D(r)=\sum_{i}^{N_{\mathrm{d}}} \mathrm{d} d_{i}(r, \Delta r)$,

the number of data-random pairs

$D R(r)=\sum_{i}^{N_{\mathrm{d}}} \mathrm{d} r_{i}(r, \Delta r)$,

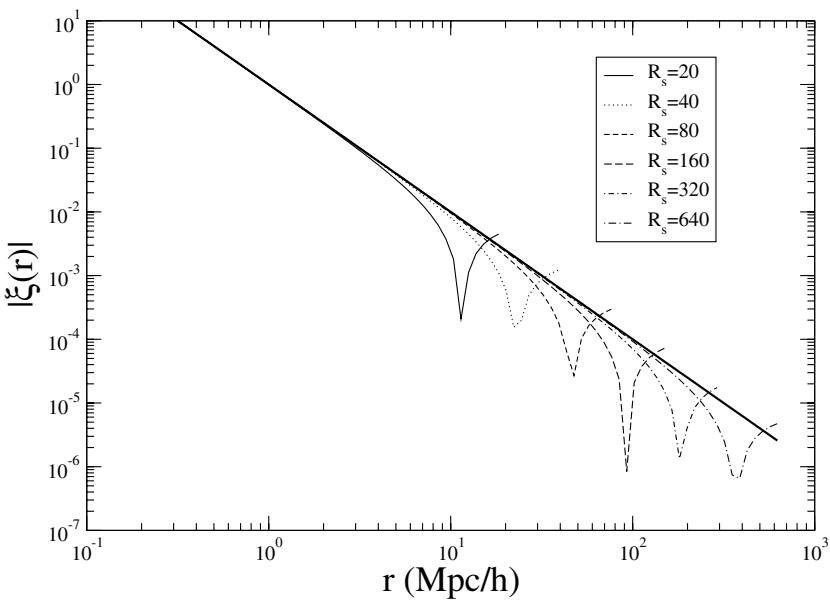

Fig. 12. Absolute value of the estimation of the correlation function $\xi(r) \sim r^{-\gamma}$, with $\gamma=2$, by the FS estimator. The thick solid line represents the theoretical model. The condition given by the integral constraint described by Eq. (37) is taken into account: beyond the scale on which there is the break of the power-law behavior, the correlation function crosses zero and becomes negative.

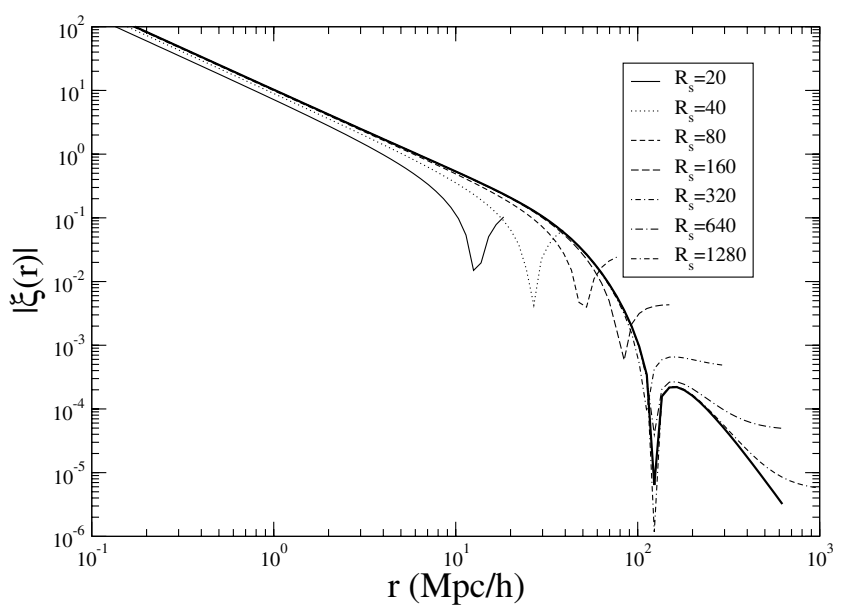

Fig. 13. Absolute value of the estimation of the correlation function of the $\Lambda \mathrm{CDM}$ model with the integral constraint described by Eq. (37). The thick solid line represents the theoretical model.

the number of random-random pairs

$R R(r)=\sum_{i}^{N_{\mathrm{r}}} r r_{i}(r, \Delta r)$

where $N_{\mathrm{d}}$ is the number of data points, $N_{\mathrm{r}}$ the number of random points, which are Poisson distributed, $\mathrm{d} d_{i}(r, \Delta r), \mathrm{d} r_{i}(r, \Delta r)$, and $r r_{i}(r, \Delta r)$ are respectively the numbers of data-data, datarandom, and random-random pairs in the shell of radius $r$ and thickness $\Delta r$ around the $i$ th center.

The DP estimator is defined as (Davis \& Peebles 1983) ${ }^{3}$

$\xi_{\mathrm{DP}}(r)=\frac{N_{\mathrm{r}}}{N_{\mathrm{d}}-1} \frac{D D(r)}{D R(r)}-1$.

${ }^{3}$ For clarity we hereafter denote the estimator as $\xi_{X X}$ where $X X$ can be FS for the full-shell case, DP for the Davis and Peebles case, and LS for the Landy and Szalay case. We omit the $\bar{X}$ symbol that was previously introduced to mean that this is an estimator of the statistical quantity $X$. 
The LS estimator is defined as (Landy \& Szalay 1993)

$\xi_{\mathrm{LS}}(r)=\frac{N_{\mathrm{r}}\left(N_{\mathrm{r}}-1\right)}{N_{\mathrm{d}}\left(N_{\mathrm{d}}-1\right)} \frac{D D(r)}{R R(r)}-2 \frac{N_{\mathrm{r}}-1}{N_{\mathrm{d}}} \frac{D R(r)}{R R(r)}+1$.

Finally the Hamilton estimator is defined as (Hamilton 1993)

$\xi_{\mathrm{H}}(r)=\frac{N_{\mathrm{r}} N_{\mathrm{d}}}{\left(N_{\mathrm{r}}-1\right)\left(N_{\mathrm{d}}-1\right)} \frac{D D(r) R R(r)}{D R^{2}(r)}-1$.

\subsection{Errors}

The measurement errors of the correlation function can be determined in various ways. This first is to calculate of the error on $\overline{\xi(r)}$ in a given sample using the Poisson estimate (Ross et al. 2007)

$\sigma_{\mathrm{P}}^{2}(r)=\frac{1+\overline{\xi(r)}}{\sqrt{D D(r)}}$

The second error estimation method is the field-to-field error, which is obtained by divining the whole sample into $N$ subsamples and by computing in each of these the correlation function $\overline{\xi_{i}(r)}$ for $i=1 \ldots N$

$\sigma_{\mathrm{FtF}}^{2}(r)=\frac{1}{N-1} \sum_{i=1}^{N} \frac{D R_{i}(r)}{D R(r)}\left(\overline{\xi_{i}(r)}-\overline{\xi(r)}\right)^{2}$,

and $\overline{\xi(r)}$ is the estimation of the correlation function in the whole sample. The third method is called the jackknife estimate (Scranton et al. 2002; Zehavi et al. 2004), and the variance is estimated by

$\sigma_{\mathrm{Jack}}^{2}(r)=\sum_{i^{\prime}=1}^{N} \frac{D R_{i^{\prime}}(r)}{D R(r)}\left(\overline{\xi_{i^{\prime}}(r)}-\overline{\xi(r)}\right)^{2}$

where the index $i^{\prime}$ is used to signify that the value of the correlation function $\overline{\xi_{i^{\prime}}(r)}$ is computed each time in all sub-samples but one (the $i$ th). Finally another possibility is to divide the sample into $N$ sub-fields, to compute the average

$\overline{\xi(r)}=\frac{1}{N} \sum_{i=1}^{N} \xi_{i}(r)$

and then the variance on the average

$\sigma_{a}^{2}(r)=\frac{1}{N} \sum_{i=1}^{N} \frac{\left(\xi_{i}(r)-\overline{\xi(r)}\right)^{2}}{N-1}$.

We show in what follows that Eq. (48) is equivalent to Eq. (46) on all but the largest scales of the sample where it gives a more conservative estimation of the errors. In what follows we make use of the errors estimated by Eq. (48), which are similar to the jackknife ones (Eq. (46)). Below we discuss in detail the determination of the errors in artificial distributions and a comparison between the different methods to define them.

\section{Test on artificial distributions}

We consider a distribution of points extracted from a cosmological $\mathrm{N}$-body simulation generated in the framework of the Millennium project (Springel et al. 2005), which consists of $N=6528040$ particles in a cubic box of nominal side $L=1$ and which is one of the semi-analytic catalogs (Croton et al. 2006)

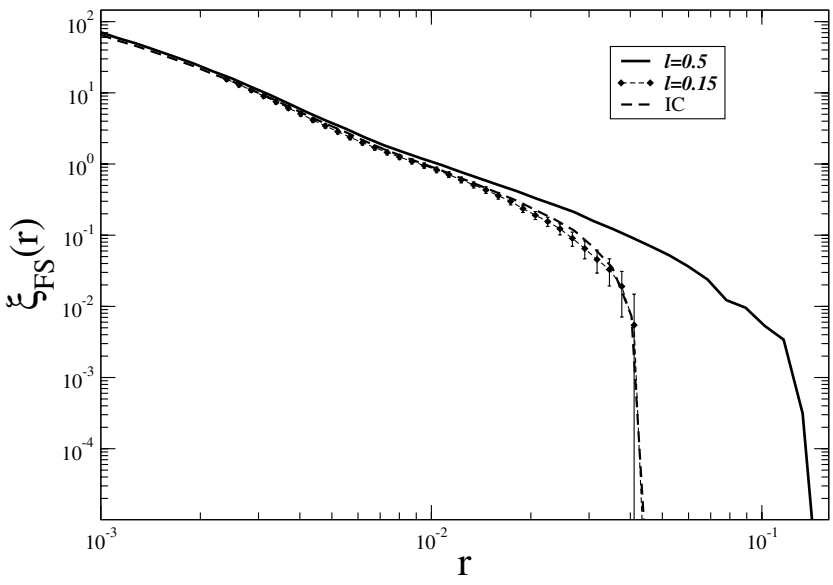

Fig. 14. Average correlation function computed by using the FS estimator in independent sub-boxes of side $\ell=0.15$, together with the prediction of Eq. (37). The solid line represents the correlation function computed by using the FS estimator in independent sub-boxes of side $\ell=0.5$, while the dotted line (IC) represents the analytical computation of the estimated correlation with the integral constraint (i.e. Eq. (37)).

constructed to produce mock galaxy samples. This distribution presents strong clustering up to a scale of $r_{0} \approx 0.01$ and then weak power-law correlations up to the sample size. We compare the results of each estimator in the sub-boxes of varying size with the determination of the FS estimator in the box of side $L=1$, which we take as a reference. In principle, one would like to have a theoretical prediction to compare with however due to the effect of the formation of non-linearities and to the sampling used to produce these distributions, one does not have a simple way to compute the theoretical correlation function. This is the reason why we have chosen the correlation function computed in the entire box as a reference. In addition, for all statistical quantities considered, we limit our analysis to the scale $R_{\mathrm{s}}=0.2$ in order to minimize the effects of the finite sample size. In what follows we report the results by using the field-to-field average quantities and variance (i.e. Eqs. (47) and (48)) which we find to be the most conservative error determinations. Below we also present a discussion of the different errors determinations.

\subsection{Cubic samples}

We have divided the box of side $L=1$ into $N_{\mathrm{f}}$ non overlapping sub-boxes of side $\ell=0.05,0.1,0.15,0.2,0.25$ and computed the correlation function in each of the sub-boxes. Note that the number sub-boxes over which the calculations are performed is taken to be constant independently of their size and $N_{\mathrm{f}}=16$. The determination of the correlation function by using the FS estimator is shown in Fig. 14. The main difference between the estimated correlation function and the "true" one is due to the integral constraint. This can be shown by the comparison of the estimated correlation function with that computed by using Eq. (37), which describes the effect of the integral constraint.

In Fig. 15 we compare the determinations of the correlation function by the FS estimator in sub-boxes of different sizes. One may note that the effect of the integral constraint, for what concerns the amplitude of the estimated correlation function, is important for the sub-samples with $\ell \leq 0.1$, because in this case the distribution is strongly non-linear inside the sample, so the determination of the sample density strongly depends on the sample size. This is shown by both a smaller amplitude and a narrower range of distance scales over which the correlation function is 


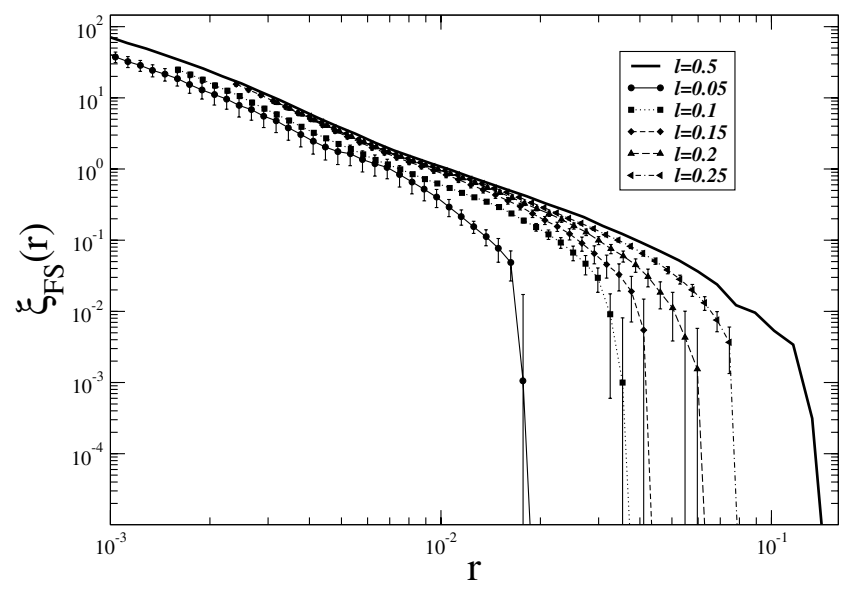

Fig. 15. Average correlation function computed by using the FS estimator in independent sub-boxes of side $\ell=0.05,0.1,0.15,0.2,0.251$. The solid line represents the correlation function computed by using the FS estimator in independent sub-boxes of side $\ell=0.5$.

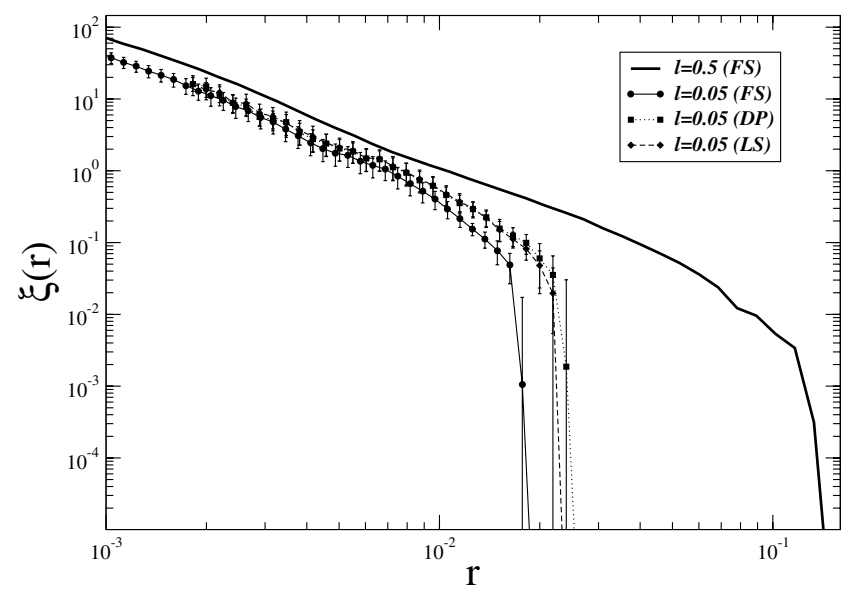

Fig. 16. Average correlation function by using the FS, LS, and DP estimator in independent sub-boxes of side $\ell=0.05$. The solid line represents the correlation function computed by using the FS estimator in independent sub-boxes of side $\ell=0.5$.

positive. The break in the positive behavior occurs at a distance scale of order $\ell$ independently of the amplitude of the correlation function. This is again a finite-size effect that can be easily understood as coming from the integral constraint.

To summarize there are two distinct effects: (i) the amplitude of the estimated correlation function strongly depends on the sample size when the distribution exhibits strong clustering and (ii) the artificial break of the positive correlations is dependent of the sample size.

In Figs. 16, 17, we compare the FS, DP and LS estimators. One can see that the DP and LS estimators are biased by a similar effect to the FS estimator, due to the integral constraint, although the break in the power-law behavior seems to occur on slightly larger scales than for the FS estimator. This difference can be attributed to the LS and DP estimators implicitly using the estimations of the average density on scale $\ell$ instead of on scale $\ell / 2$ as the FS estimator. To clarify this point in the next section we present some other tests that have been tuned to explore this effect.

In Fig. 18 we compare the LS and Hamilton estimators. We confirm the results of Kerscher et al. (2000) that the Hamilton and LS estimators give indistinguishable results inside the error bars, and thus we focus on the former.

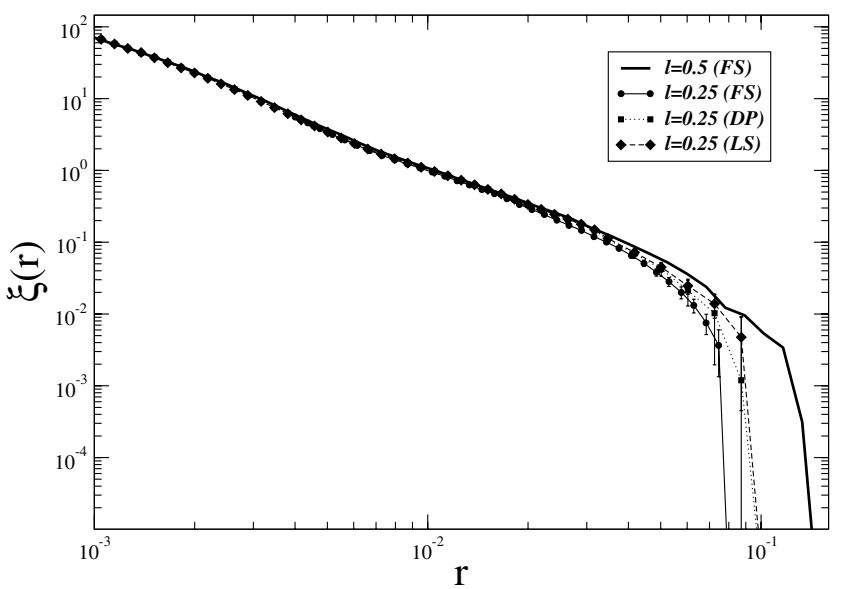

Fig. 17. Average correlation function computed by using the FS, LS, and DP estimator in independent sub-boxes of side $\ell=0.25$. The solid line represents the correlation function computed by using the FS estimator in independent sub-boxes of side $\ell=0.5$.

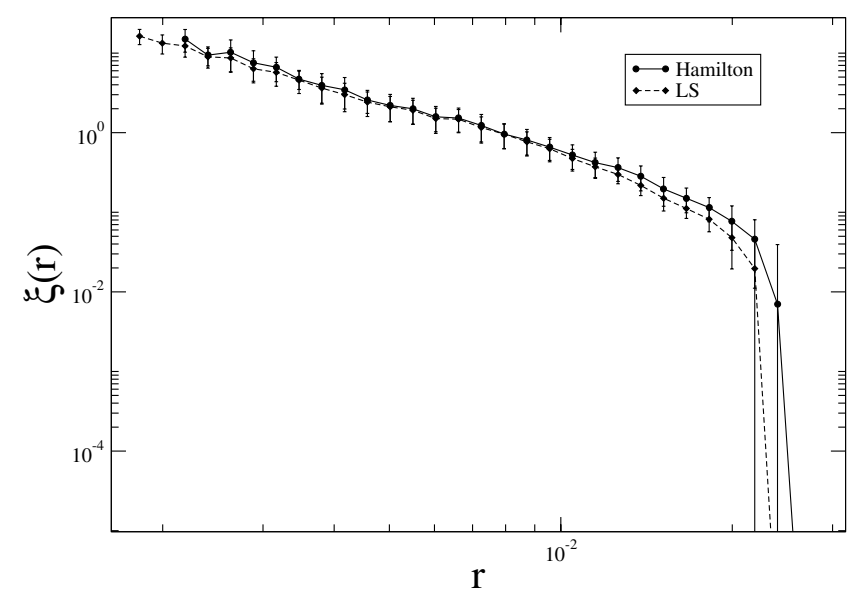

Fig. 18. Average correlation function by using the LS and Hamilton estimator in independent sub-boxes of side $\ell=0.05$.

In Figs. 19, 20 we show the determinations of the average correlation function computed by using the LS and DP estimators in independent sub-boxes of side $\ell=0.05,0.1,0.15,0.2$, 0.25: dependencies on the sample finite size of the amplitude and of the break are still present as for the FS estimator, and analogously to this case, they can be understood as an effect of the integral constraint.

\subsection{Slices}

We have seen that the estimation of the correlation function depends on the sample size, that up to now has been considered to be a simple geometrical shape such as a sphere or a cubic box. In order to investigate a situation closer to real observations, we have constructed several sub-samples of the original distribution in the following way. We placed the observer placed in the center of the box $(0.5,0.5,0.5)$ and we have identified a sphere of radius 0.5 centered on that point. In addition we have considered the spherical coordinates $\alpha, \delta, r$ of the distribution points with respect to such a center, where $0 \leq \alpha \leq 2 \pi,-\pi / 2 \leq \delta \leq \pi / 2$ and $0 \leq r \leq 0.5$. It is now possible to construct several sub-samples, that have a certain depth $R_{\text {depth }} \leq 0.5$ and specific cuts in $\alpha$ and $\delta$. In general the solid angle of a portion of a sphere is

$\Omega=\Delta \alpha \times \Delta \mu$, 


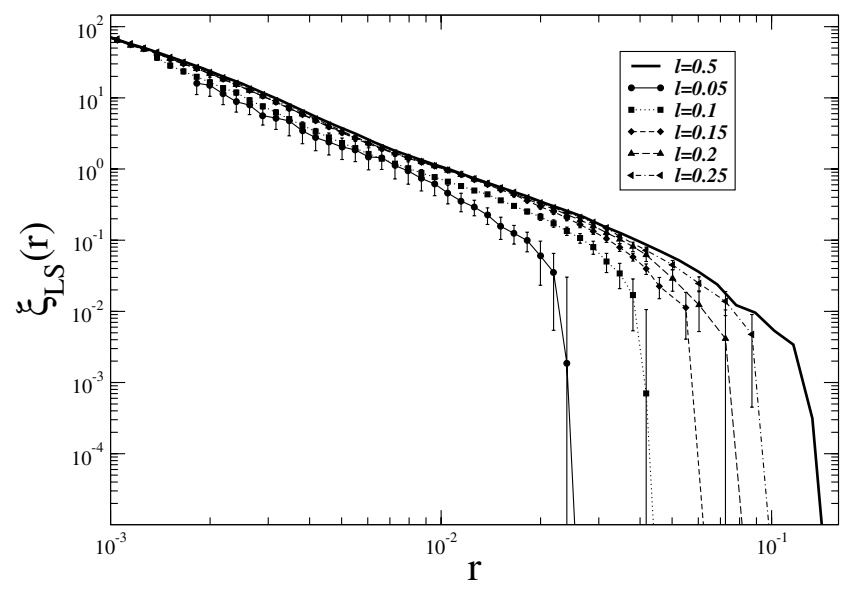

Fig. 19. Average correlation function computed by using the LS estimator in independent sub-boxes of side $\ell=0.05,0.1,0.15,0.2,0.25$. The solid line represents the correlation function computed by using the FS estimator in independent sub-boxes of side $\ell=0.5$.

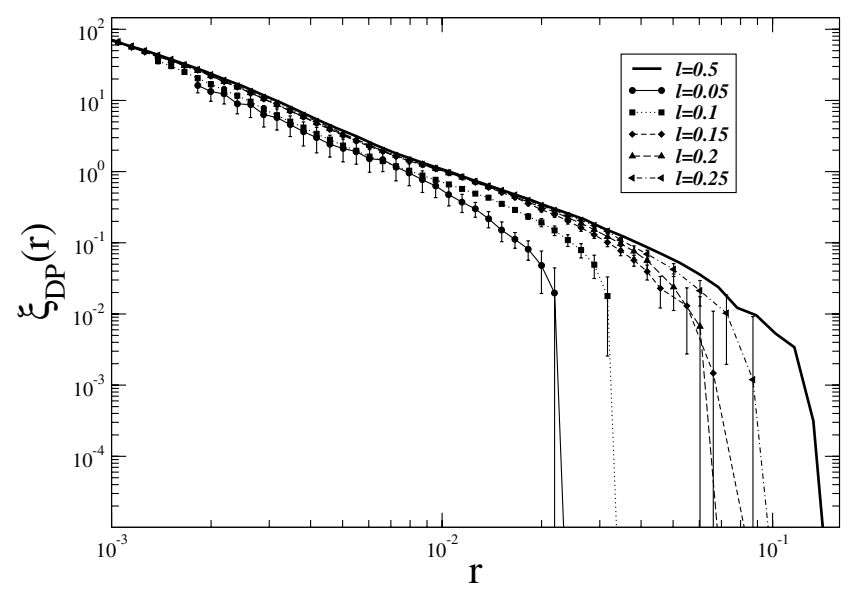

Fig. 20. Average correlation function computed by using the DP estimator in independent sub-boxes of side $\ell=0.05,0.1,0.15,0.2,0.25$. The solid line represents the correlation function computed by using the FS estimator in independent sub-boxes of side $\ell=0.5$.

where $\Delta \alpha=\alpha_{2}-\alpha_{1}$ with $\alpha_{1}, \alpha_{2}$ the limits in right ascension defining the angular region and $\Delta \mu=\sin \left(\delta_{2}\right)-\sin \left(\delta_{1}\right)$, with $\delta_{1}, \delta_{2}$ the limits is declination defining the angular region. We choose $\Delta \mu=2$, i.e. $\delta_{1}=-\pi / 2$ and $\delta_{2}=\pi / 2$ and $\Delta \alpha=$ const. In such a way, we constructed $N_{\mathrm{f}}$ independent spherical slices with a constant solid angle and same geometry. The number of slices is thus $N_{\mathrm{f}}=2 \pi / \Delta \alpha$ : we took $N_{\mathrm{f}} \leq 30$. We then computed the LS and DP estimators and their field-to-field variance (Eq. (48)).

In Figs. 21-23 we show the average correlation function computed by using the LS and DP estimators in $N_{\mathrm{f}}=30$ angular slices with $\Delta \alpha=0.0063,0.013,0.063$ respectively. One sees that the LS and DP estimators are very similar, although the LS estimator extends to fairly large scales. The amplitude in this case corresponds to the expectation value for the FS estimator in a box of side $R_{\text {depth }}=0.05$, which is about ten times larger than the radius of the maximum sphere fully included in the sample volume.

By comparing (see Figs. 24, 25) the FS, LS, and DP estimators computed in angular slices with $\Delta \alpha=0.0063,0.013,0.063$ one can see that the amplitude increases slightly by choosing a larger solid angle, and the range of scales where one may estimate the correlation function also increases when $\Delta \alpha$ increases. The exact location of the break in the power-law behavior and

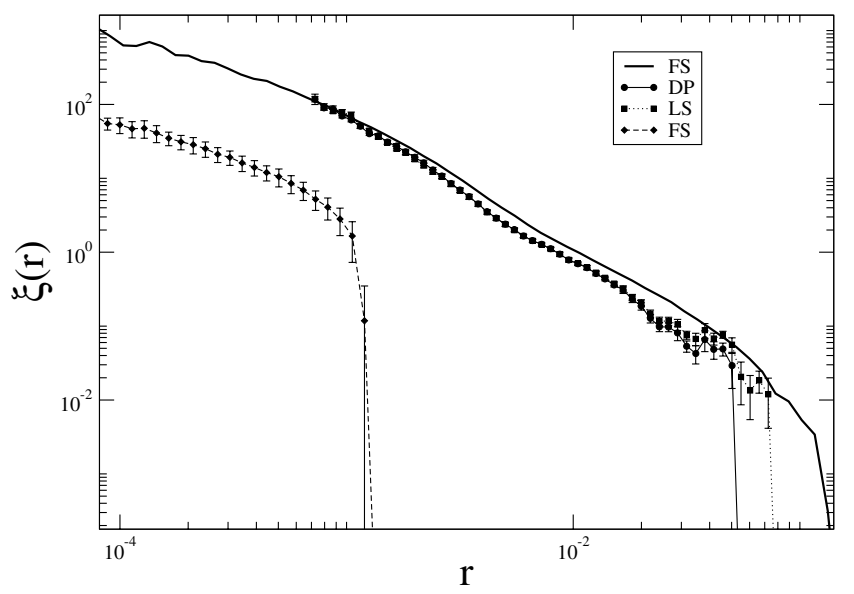

Fig. 21. Average correlation function computed by using the FS, LS and DP estimators in $N_{\mathrm{f}}=30$ angular slices with $\Delta \alpha=0.0063$. The solid line represents the correlation function computed by using the FS estimator in independent sub-boxes of side $\ell=0.5$.

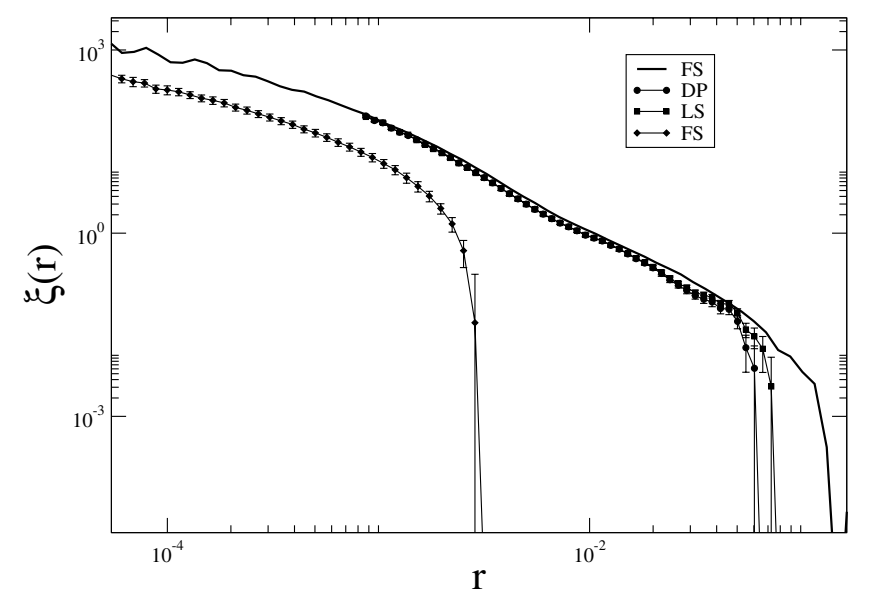

Fig. 22. Average correlation function computed by using the FS, LS and DP estimators in $N_{\mathrm{f}}=30$ angular slices with $\Delta \alpha=0.013$. The solid line represents the correlation function computed by using the FS estimator in independent sub-boxes of side $\ell=0.5$.

the value of the amplitude are in agreement with a value of $R_{\text {depth }}$ in integral constraint on the order of the sample depth $\ell$ and not of the radius of the maximum sphere fully enclosed as for the case of the FS estimator.

Finally in Fig. 26 we show the average behavior of the LS estimator in $N_{\mathrm{f}}=30$ angular slices with $\Delta \alpha=0.063$ and with a varying depth of the sample $R_{\text {depth }}=0.1,0.2,0.5$. The dependence finite of the amplitude and of the scale at which the break in the power-law behavior occurs on the finite size of the sample is clear. This represents an interesting test of the galaxy data as we discuss below.

\subsection{Determination of the errors}

In Fig. 27 we show the behavior of the errors computed by Eqs. (44)-(46) and Eq. (48). Note that errors determined by the jackknife method, Eq. (46), are approximately the same as the ones computed by the field-to-field fluctuations of Eq. (48), except on small scales where the jackknife method is more efficient by giving smaller fluctuations (see discussion in Scranton et al. 2002; and Zehavi et al. 2002). On the other hand, the jackknife error is greater than the two other estimators, Eqs. (44), (45) (see also Ross et al. 2006). Apart from the small difference on small 


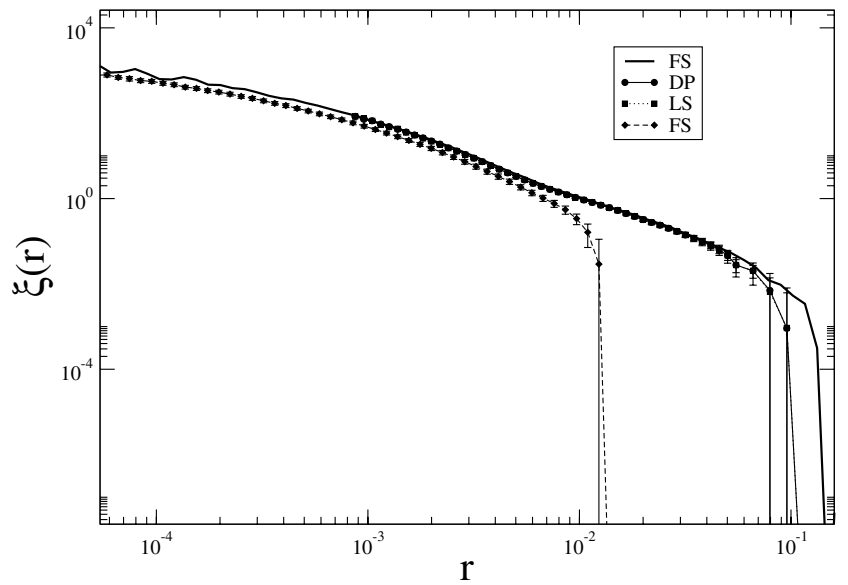

Fig. 23. Average correlation function computed by using the FS, LS and DP estimators in $N_{\mathrm{f}}=30$ angular slices with $\Delta \alpha=0.063$. The solid line represents the correlation function computed by using the FS estimator in independent sub-boxes of side $\ell=0.5$.

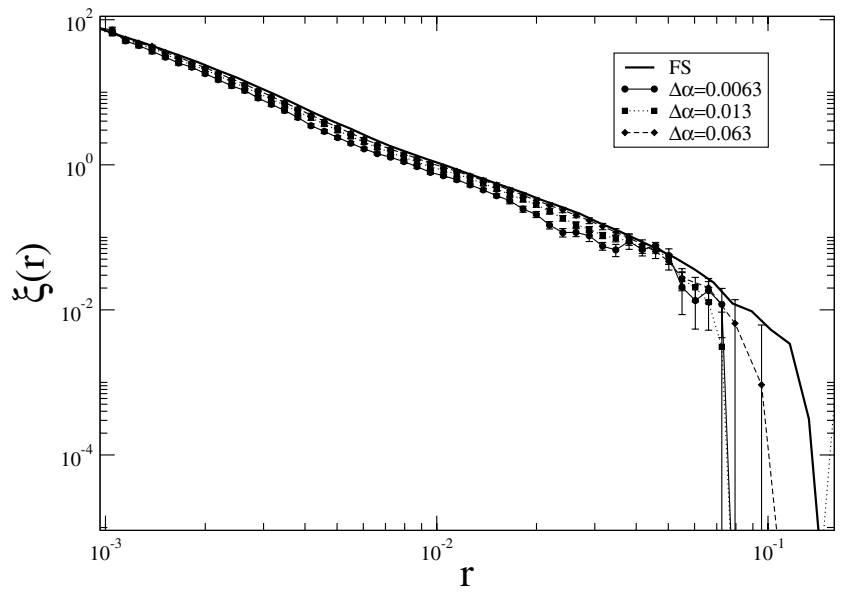

Fig. 24. Average correlation function computed by using the LS estimator in $N_{\mathrm{f}}=30$ angular slices with $\Delta \alpha=0.0063,0.013,0.063$. The solid line represents the correlation function computed by using the FS estimator in independent sub-boxes of side $\ell=0.5$.

scales between Eqs. (46)-(48) the former are larger on scales comparable to the sample size, and give the more conservative estimation of the fluctuations.

\subsection{Summary and discussion}

We have studied the finite size dependence of the estimated twopoint correlation function by considering three different estimators the FS, the DP and the LS. We considered the case of a point distribution presenting, on large enough scales in the sample, weak $(\xi(r)<1)$ power-law correlations. We performed a series of tests to establish the role of the biases due to the integral constraint. This is the principal systematic effect that affects the behavior of the estimated correlation function on large scales, independently of the particular estimator considered. Let us briefly discuss our main results.

We first considered the determination of the correlation function in the cubic sub-sample of size $\ell<L=1$, where $L$ is the whole box size. We constructed our estimation as an average over $N_{\mathrm{f}}$ disjointed sub-boxes. We have studied the behavior of the FS estimator as a function of the size $\ell$ of the sub-boxes, finding a clear dependence on the sample finite size for both the

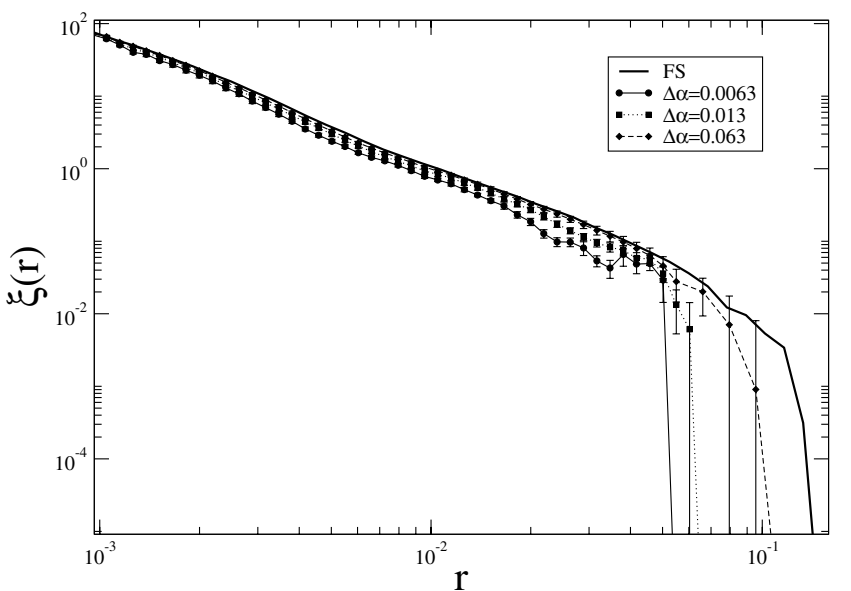

Fig. 25. Average correlation function computed by using the DP estimator in $N_{\mathrm{f}}=30$ angular slices with $\Delta \alpha=0.0063,0.013,0.063$. The solid line represents the correlation function computed by using the FS estimator in independent sub-boxes of side $\ell=0.5$.

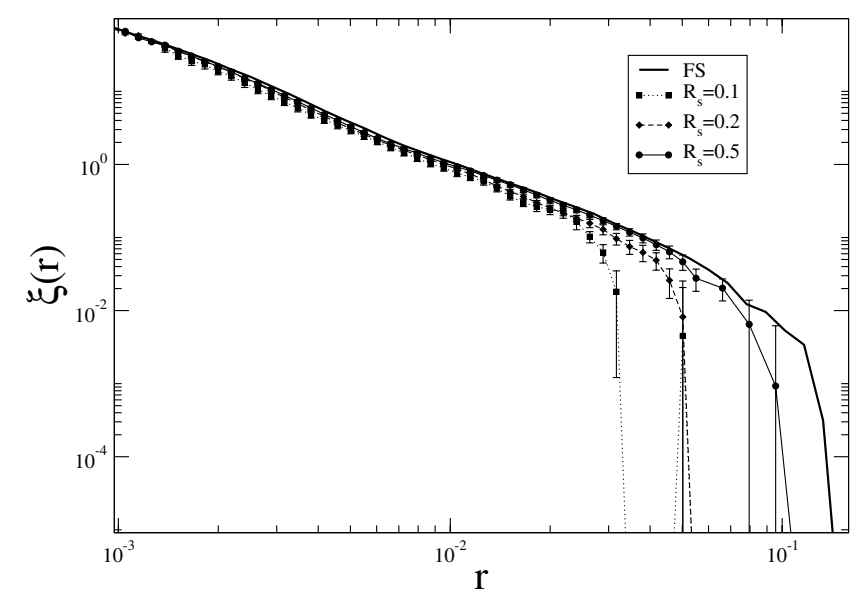

Fig. 26. Average correlation function computed by using the LS estimator in $N_{\mathrm{f}}=30$ angular slices with $\Delta \alpha=0.063$ and with a varying depth of the sample $R_{\text {depth }}=0.1,0.2,0.5$. The solid line represents the correlation function computed by using the FS estimator in independent sub-boxes of side $\ell=0.5$.

amplitude (for small $\ell$ ) and the length scale $r^{*}$ characterizing the break in the power-law behavior, beyond which the correlation function becomes negative. In agreement with a simple analytical study of the problem discussed in the previous section we found that $r^{*} \sim \ell / 2$. A similar situation occurs for the LS and DP estimators even though $r^{*} \sim \ell$ in this case. We note that the LS and DP estimators give very similar results over the whole range of scales.

To understand the spatial extension of the reliable measurements of two-point correlations provided by different estimators in more detail we considered samples with geometry that is more similar to the case of real galaxy samples; namely we considered a sphere around the central point in the box of size $L$ and divided it into $N_{\mathrm{f}}$ sub-samples with the same solid angle $\Omega$. We also considered subsequent cuts in the depth $\ell<L$. We found that the length-scale $r^{*}$ shows a dependence on $\Omega$ and it typically reaches a value on the order of a fraction of $\ell$, which is larger than the scale $R_{\mathrm{s}}$, up to which the FS estimator can be applied and which is of the order of the radius of the maximum sphere fully enclosed in the sample volume. We then measured that the scale $r^{*}$ has a strong dependence on the value of $\ell$ such as for the case of the simple cubic volumes considered in the previous test. 


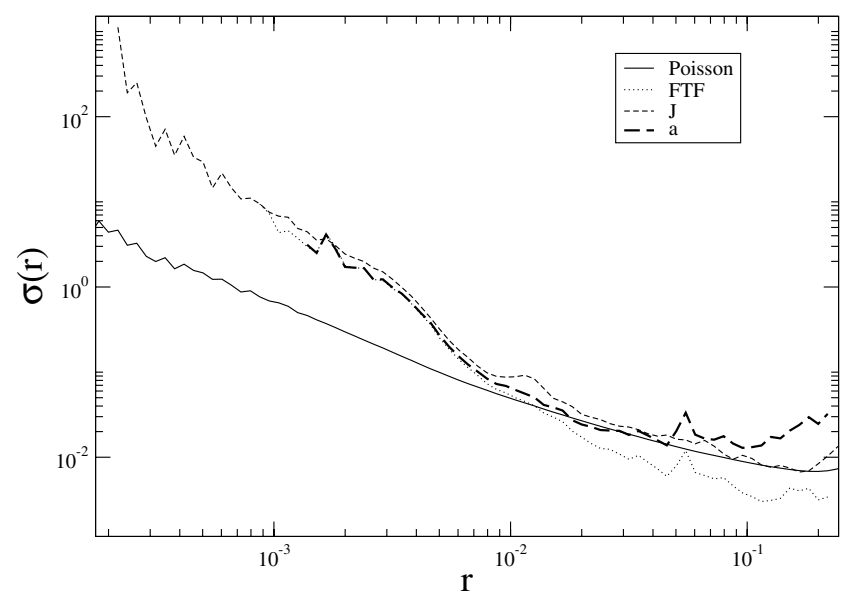

Fig. 27. Errors in the estimation of the correlation function determined by Eqs. (44) (Poisson), (45) (FTF), (46) (J), and (48) (a).

It is important to note that the tests discussed here were performed on a distribution which becomes uniform well inside the sample size. The above considerations on the performance of the various estimators can be easily verified for other distributions that satisfy the property of becoming uniform well inside a given sample and that show different correlation properties on large scales. However, the situation is rather different for the case in which a distribution exhibits strong clustering inside a given sample without a clear crossover toward a uniform distribution. In this case the best estimator is the most conservative one; i.e., the FS estimator as the estimation of the sample density is certainly biased on any scale as long as the distribution is characterized by strong non-linear clustering (see discussion in, e.g. Gabrielli et al. 2004, for the treatment of the strongly correlated case).

This situation creates a serious warning about the determination of the correlation on large scales in a given sample. If an estimator correlation function presents a break in, for example, the power-law behavior on a certain scale, the crucial test to be performed is to check whether this is a finite size or whether it is a true break. This situation is especially relevant for CDM-type correlations, for which the correlation function, according to theoretical models, should present a break from the small-scale power-law correlation on a scale of about $124 \mathrm{Mpc} / \mathrm{h}$. We will come back on this point in the conclusion.

Finally, we considered different determinations of the errors of the estimators of the two-point correlation function. The more conservative way to estimate errors consists in the computation of the correlation function in disjointed regions and then to compute the average and the variance on the average: this method is less efficient than the jackknife method on small scales but gives similar results to that on large scales.

\section{Conclusions}

We have considered the real-space properties of CDM density fields, focusing in detail on a particular variant known as the $\Lambda C D M$ model. It is well known that the power spectrum typically has a behavior $P(k) \sim k^{m}$ with $-1<m \leq-3$ for large wavelengths $k>k_{\mathrm{c}}$, and $P(k) \sim k$ at smaller wavelengths $k<k_{\mathrm{c}}$. We discussed that, correspondingly, the two-point correlation function approximately shows a positive power-law behavior $\xi(r) \sim r^{-2}$ on small scales $r<r_{\mathrm{c}} \approx k_{\mathrm{c}}^{-1}$ and a negative power-law behavior $\xi(r) \propto-r^{-4}$ on large scales $r>r_{\mathrm{c}}$, where the zero-crossing occurs at about $r_{\mathrm{c}} \approx 124 \mathrm{Mpc} / \mathrm{h}$ in the model considered. We discussed the fact that, globally, a system with this type of correlations belongs to the category of superhomogeneous distributions, which are configurations of points that are more ordered than a purely uncorrelated (Poisson) distribution. Correspondingly fluctuations are depressed with respect to the Poisson case, and the normalized mass variance, for instance, decays faster $\left(\sigma^{2}(r) \sim r^{-4}\right)$ than for the Poisson case $\left(\sigma^{2}(r) \sim r^{-3}\right)$. The condition of super homogeneity is expressed by the condition that $P(k) \rightarrow 0$ for $k \rightarrow 0$, or alternatively that

$\int_{0}^{\infty} \xi(r) r^{2} \mathrm{~d} r=0$

Following the work of Durrer et al. (2003) we pointed out that the above condition is broken when one samples the distribution, as for example when the simplest biasing scheme of correlated Gaussian fields (introduced by Kaiser 1984) is applied. This is particularly important for the behavior of the power spectrum for $k<k_{\mathrm{c}}$, which, under biasing, remains constant instead of going as $P(k) \sim k$. The correlation function at large scales $r>$ $r_{\mathrm{c}}$ is instead expected to be linearly amplified with respect to the original one of the whole matter field. Thus the large-scale negative tail $\xi(r) \sim-r^{-4}$ is the main feature that one would like to detect in order to test theoretical models.

Given the fact that when $\xi(r)$ becomes negative, it is characterized by a very small amplitude, determining the negative power-law tail is challenging. We discussed the fact that, at first approximation in a real measurement, one may treat the system as having positive correlations on small scales with an exponential cut-off on the scale $r_{\mathrm{c}}$ and then it becomes uncorrelated (a situation which can be regarded as an upper limit to the presence of anti-correlations). This implies that, for $r_{\mathrm{c}}>124 \mathrm{Mpc} / \mathrm{h}$, galaxy distribution should not present any positive correlation. Whether this behavior is compatible with the existences of structures around $200 \mathrm{Mpc} / \mathrm{h}$, or more is an open problem to be addressed in studies of forthcoming galaxy catalogs.

More in detail, one of the most basic results (see e.g., Peebles 1980) for self-gravitating systems, treated using perturbative approaches to the problem (i.e. the fluid limit), is that the amplitude of small fluctuations grows monotonically in time, in a way that is independent of the scale. This linearized treatment breaks down on any given scale when the relative fluctuation on the same scale becomes of order unity, signaling the onset of the "non-linear" phase of gravitational collapse of the mass in regions with the corresponding size. If the initial velocity dispersion of particles is small, non-linear structures start to develop on small scales first and then the evolution becomes "hierarchical", i.e., structures build up on successively larger scales. Given the finite time from the initial conditions to the present day, the development of non-linear structures is limited in space, i.e., they cannot be more extended than the scale on which the linear approach predicts that the density contrast becomes of order unity at the present time. This scale is fixed by the initial amplitude of fluctuations, constrained by the cosmic microwave background anisotropies (Spergel et al. 2007), by the hypothesized nature of the dominating dark matter component and its correlation properties. According to current models of CDM-type the scales at which non-linear clustering occurs at the present time (of order $10 \mathrm{Mpc}$ ) are much smaller than the scale $r_{\mathrm{c}} \approx 124 \mathrm{Mpc} / \mathrm{h}$ (see e.g. Springel et al. 2005). Thus the region where the superhomogeneous features should still be in the linear regime, allowing a direct test of the initial conditions predicted by early universe models. The scale $r_{\mathrm{c}}$ marks the maximum extension of positively correlated structures: beyond $r_{\mathrm{c}}$ the distribution must 
have been anti-correlated since the beginning, as there was no time to develop other correlations. The presence of structures reported both by observations of galaxy distributions (like the Sloan Great Wall - see Gott et al. 2005), by the detection of dark matter distributions (see e.g. Massey et al. 2007) and by the large void of radius $\sim 140 \mathrm{Mpc}$ identified by Rudnick et al. (2007), is maybe indicating that positive correlations extend well beyond $r_{\mathrm{c}}$.

We have discussed that an important finite size effect must be considered when estimating the correlation function and may mimic a break in the power-law behavior similar to the ones of CDM models on a scale near $r_{\mathrm{c}}$. This is related to the effect of the integral constraint in the estimators, namely the fact that the sample average, estimated in a finite sample, differs from the ensemble average, and thus can be dependent of the finite-size of a given sample. This situation occurs when correlations (weak or strong) extend to larger scales than the sample size.

For these reasons, and to study the two-point correlation function in real galaxy samples when its amplitude becomes smaller than unity, it is crucial to check whether the break in the power-law behavior is dependent of the sample finite size, by choosing samples with different depths. In this perspective the assessment of the reality of the break of the two-point correlation function is the main observational point to be considered. Once this is clarified, other features should be considered, as for the example the so-called baryonic bump, which is a very small perturbation to the overall shape of the correlation function on scales near the zero-point $r_{\mathrm{c}}$. We will present a detailed analysis of the correlation properties of galaxy distribution in the SDSS catalog, considering specific tests for finite-size effects in the determination of the correlation function, in a forthcoming paper.

Acknowledgements. We are grateful to Y. Baryshev, A. Gabrielli, M. Joyce, B. Marcos and L. Pietronero for useful discussions and comments. F.S.L. thanks the MIUR-PRIN05 project on "Dynamics and thermodynamics of systems with long range interactions" for financial support.

\section{References}

Benoist, C., Maurogordato, S., da Costa, L. N., et al. 1996, ApJ, 472, 452 Bond, J. R., \& Efstathiou, G. 1984, ApJ, 285, 45

Croton, D. J., Springel, V., White, S. D. M., et al. 2006, MNRAS, 365, 11 Davis, M., \& Peebles, P. J. E. 1983, ApJ, 267, 46

Davis, M., Meiksin, A., Strauss, M. A., et al. 1988, ApJ, 333, L9

Durrer, R., Gabrielli, A., Joyce, M., \& Sylos Labini, F. 2003, ApJ, 585, L1

Eisenstein, D. J., Zehavi, I., Hogg, D. W., et al. 2005, ApJ, 633, 560

Eisenstein, D. J., Seo, H.-J., Sirko, E., \& Spergel, D. N. 2006 [arXiv: astro-ph/0604362]

Gabrielli, A., Joyce, M., \& Sylos Labini, F. 2002, Phys. Rev. D, 65, 083523

Gabrielli, A., Sylos Labini, F., Joyce, M., \& Pietronero, L. 2004, Statistical physics for cosmic structures (Springer Verlag)

Gott, J. R. III, Juric, M., Schlegel, D., et al. 2005, ApJ, 624, 463

Kaiser, N. 1984, ApJ, 284, L9

Kerscher, M. 1999, A\&A, 343, 333

Kerscher, M., Szapudi, I., \& Szalay, A. S. 2000, ApJ, 535, 13

Hamilton, A. J. S. 1993, ApJ, 417, 19

Hewett, P. C. 1982, MNRAS, 201, 867

Landy, S. D., \& Szalay, A. 1993, ApJ, 412, 64

Massey, R., Rhodes, J., Ellis, R., et al. 2007, Nature, 445, 286

Padmanabhan, T. 1993, Structure formation in the universe (Cambridge: Cambridge University Press)

Park, C., Vogeley, M. S., Geller, M., \& Huchra, J. 1994, ApJ, 431, 569

Peebles, P. J. E. 1980, The Large-Scale Structure of the Universe (Princeton University Press)

Ross, N. P., da Angela, J., \& Shanks, T. 2007, MNRAS, 381, 573

Rudnick, L., Brown, S., \& Williams, L. R. 2007, ApJ, in press [arXiv:0704.0908v2]

Scranton, E., Johnston, D., Dodelson, S., et al. 2002, ApJ, 579, 48

Spergel, D. N., Bean, R., Doré, O., et al. 2007, ApJS, 170, 377

Springel, V., White, S. D. M., Jenkins, A., et al. 2005, Nature, 435, 629

Sylos Labini, F., Vasilyev, N. L., \& Baryshev, Yu. V. 2007, A\&A, 465, 23

Tegmark, M., Blanton, M. R., Strauss, M. A., et al. 2004, ApJ, 606, 702

Totsuji, H., \& Kihara, T. 1969, PASJ, 21, 221

Vasilyev, N. L., Baryshev, Yu. V., \& Sylos Labini, F. 2006, A\&A, 447, 431

York, D. G., Adelman, J., Anderson, J. E., Jr., et al. 2000, ApJ, 120, 1579

Zehavi, I., Blanton, M. R., Frieman, J. A., et al. 2002, ApJ, 571, 172

Zehavi, I., Weinberg, D. H., Zheng, Z., et al. 2004, ApJ, 608, 16 